\title{
Index rerum et index nominum Vol. 64-100 (1939-1963)
}

\section{Confecerunt Michel Demole et Hélène Baerlocher}

\section{BASEL (Schweiz) S. KARGER NEW YORK}

$(\mathrm{A})=$ Analyses - Referate - Abstracts $(\mathrm{C})=$ Casuistique - Kasuistik - Case

report $(\mathrm{E})=$ Editorial $(\mathrm{L})=$ Livres nouveaux - Buchbespre-

chung - Book review

$(\mathrm{N})=$ Necrologia

$(\mathrm{R})=$ Revue générale - Übersichtrefe-

rat - Review $(\mathrm{S})=$ Sociétés - Gesellschaftsbericht -

Society transactions $(\mathrm{T})=$ Page de thérapeutique - Seite der

Therapie

Les chiffres en ítalíque correspondent au volume, les chiffres droits correspondent à la page; Гannée n'est jamais indiquée.

Les travaux orígínaux sont caractérisés par deux nombres, sans lettre entre parentheses.

Die kursív gesetzten Zahlen entsprechen dem Band, die geradestehenden Zahlen ent-sprechen der Seite; die Jahreszahl ist nirgends angegeben.

Die Origínalarbeíten sind durch zwei Zahlen gekennzeichnet, ohne in Klammern gesetzte Buchstaben.

The numbers in italics correspond to the volume; the roman numbers correspond to the page; the year is never indicated.

The original papers are indicated by two numbers, without a letter in brackets.

Index rerum ad vol. 64-100

Abdomen (v. Digestio, Gastroenterolo-

gia, Peritoneum) -, diagnostics urgents 94: 331 (L) -, Differentialdiagnose kugeliger Gebilde

89: 205 -, Frühdiagnose des akuten - 94:128 (L) -, sémiologie chirurgicale 99: 74 (L) -, urographie descendante dans le diagnostic de $\Gamma$ - aigu 86: 53 (E)

Abdominal

-, chirurgie - preventive 82: 306 (E) -, Erkrankungen, Symptomatologie

72: 125 -, Operationen, gastroskopische Beob-

achtungen 67: 132 -, syndromes - aigus pseudo-chirurgi-

caux 97: $55(\mathrm{~L})$

Absorption durch Zwölffingerdarm

65: 72 Accidents, médecine des - et ulcères gas-

tro-duodénaux 67: 1

A. C. T. H. (v. Cortisone)

87: 23

$$
\text { in differential diagnosis of jaundice }
$$

-, treatment in acute hepatitis $83: 148$,

313 (T); 87: 380 -, - of postoperative pancreatitis 93: 129

und Vitamin-C, Wirkung auf Magen-

sekretion nach Histamin 77: 299

Adénosin-tri-phosphorique, acide -, action sur la cirrhose au CC14 et sur la capacité régénératrice du foie 81: 153

Adhaesiones

- $\quad$ abdominales, traitement paradoxal

(operation de Noble) 77: 303 (E)

-, surgery of peritoneal $-92: 173$

Adrenal, glands: v. Surrenal Adrenalin, discharges in pathogenesis of

gastroduodenal diseases 73: 219 Aging, nutrition in relation to - 80: 193

Alcool

est-il un aliment? 82: 177 (E)

Test als Leberfunktionsprüfung 74: 341

Alimentatio

64: $251(\mathrm{R}) ; 65: 300(\mathrm{~A}), 379(\mathrm{~A})$;

66: $52(\mathrm{~A}), 113(\mathrm{~A}) ; 67: 104(\mathrm{~A}) ; 68$ :

322 (A); 69: 334 (A); 70: 350 (A)

71: $373(\mathrm{~A}) ; 73: 202(\mathrm{~A}) ; 79: 380(\mathrm{~L})$; 
85: 64 (L); 94: 198 (L) Alimenta 70: 352 (A); 71: 375 (A) -, tables de composition 74: 187 (L) -, chemical composition 69: 119 (L) -, microbial -, food poisoning, therapy

96: 395 (T) Alimentäre Idiosynkrasie in konstitu-

tioneller Beleuchtung 66: 121 -, Alkalosis 89: 335 (C) -, insuffisance 76: 252 (L) -, regime - d'un groupe de dix families

de condition modeste 68: 340 (L) Alimentation, cholesterol, activité physique et coronarites 99: 59 (E) Ernährung, Kolloquium über parente-

rale - 98: 64 (L) -, und Darmflora 59: 60 (L) -, Bedingungen, Diastasewerte im Urin

und Blutserum unter bestimmten -

66: 12

Ausnützung 86: 90

AUergie

75: $55(\mathrm{~L}) ; 82: 370(\mathrm{~A})$

- $\quad$ digestive $98: 128(\mathrm{~L})$

276

Index rerum ad vol. 64-100

Allergie

-, le gastro-entérologue devant $\Gamma$ - ali-

mentaire 100: 259 (E) -, Nahrungsmittel -, diätetische Be-

handlung 68: 112 (L)

nutritive 64: $234(\mathrm{R})$

AUergische Entzündungen im Diinndarm

97: 156

Gastritis, akute pseudotumoröse 97: 65

Krankheiten, Histidinbehandlung 71: 233 (R)

Krankheitszustände, Hypercitricämie 69: 204 (R)

-, Hefe- und Schimmelpilze - mit gastro-intestinaler Reizantwort 89: 153

Almonds, in ulcer therapy 86: 505

Aluminium, Dihydroxy -, antacid effect and use in peptic ulcer 91: 214 (T)

Amibes: v. Parasiti

Ammoniémie et affections hépatiques 86: 421; 87: 192

Amoeba: v. Parasiti

Amphétaminomanie 98: 388 (E)

Anaemia (v. Haemorrhagia, Sanguis)

- $\quad$ gastric lesion in - 79: 329

Anaemia perniciosa

-, megaloblastic and subacute, combined degeneration from tuberculous disease of the small intestine 79: 342

-, -, following operations on intestine 79: 338

- $\quad$ et predispositions aux cancers diges

tifs (espagnol) 86: 511

- , culture in vitro de moëlle osseuse

70: 171 -, deformity of lower end of æsophagus

and adjacent part of stomach 79: $376-$, Genese 64: 19

-, hämopoetischen Stoífe 66: 288 -, histologische und zytologische Unter-

suchungen 96: 126

de Biermer, polypose gastrique et

cancer d'estomac 98: 170

-, Behandlung 65: 309 (L) -, hypochrome nach Magenresektion und Osteomalacie 66: 182 bei Kyphoskoliose 68: 197

Anämische Fieber 73: 56, 173, 248

Androgene, Leberschädigung durch -und anabole Steroide 96: 263 (T)

Antibiotica

-, accidents, casuistique 84: 334 (C)

lors d'appendicites aiguës, traite

ment abortif 89: 173

-, diarrhées graves après - 81: 238 (E) -, oral, haematological and nutritional effects 79: 327

Antrényl Duplex dans traitement ulcus 90: 39

Anus (v. Proctologia, Rectum)

- $\quad$ 68: $314(\mathrm{~A}) ; 84: 381(\mathrm{~L})$ 
-, fistules, petite chirurgie 82: 49 (L) -, hémorrhoïdes et leur traitement 76:

381 (L) -, Pruritus 73: 1

Aphtose, Erkrankungen der Mundhöhle

70: 270

traitement buccal 90: 53 (E)

Appendix vermiformis

65: 117 (A); 66: 244 (A); 68: 309 (A); 69: 214 (A); 71: 244 (A); 72: 401 (A); 75: 173 (A); 79: 171 (A); 80: 383 (A); 82: 189 (A); $84: 140$ (A); $86: 126$ (A); $94:$

326 (A) -, Appendicitis 65: 80; $92: 278$

- $\quad$ et diarrhée sanglante 95: 121 (C)

-, trouvaille radiologique 85: 40

-, sterkoraler, Appendostase als Ur-sache - 77: 360

aiguë, traitement abortif par anti-

biotiques 89: 173

Aqua

-, troubles du métabolisme du sel et -72: 77 (L)

und Elektrolytenaushalt 90: 133,

149, 204; 94: 130 (L)

-, Meer als Heilmittel 65: 310 (L)

Arteritis, Pankreasnekrosen als Folge generalisierter - 72: 81

Index rerum ad vol. 64-100

277

Arthritis: v. Rheuma Arzneimittelforschung, Fortschritte 100: 134 (L)

Ascite $\beta$ 79: 251 (A)

-, Permeabilität der Kapillärmembra-

nen in Bauchhöhle 96: 37 -, traitement par P.A.S. intrapéritonéal

90: 55 (T)

Aspirine et hemorragie digestive 71: 274 (E);96:69(L)

Association: v. Societates

Assurance-vie, gastrectomie et -86: 119 (E)

Asthma bronchialis and hiatal hernia 93: 337; 94: 351; 95: 110

Auto-antikörper und Fermentaktivität, Serumuntersuchung über - 91: 402

Azotemia in gastro-intestinal haemorrhages 84: 70 (E)

Bäder, Heil- und Krankheiten der Ver-

dauungsorgane 67: 68 (R) Banti, Syndrome de - 81: 346 (A)

Barium: v. Röntgen

-, ein neues Kontrastmittel 74: 193

- meal examination, combined chole-

cystography 89: 330 -, lavement (technique) 92: 275

Bauchspeicheldrüse: v. Pancreas Begutachtung, internistische - in Krankheiten des Magendarmkanals und der Gallenwege 88: 72 (L)

Bellum

-, Guerre, alimentation de - 69: 105 (A); 70: 356 (A)

,-- et maladies digestives $68: 247$

,-- et recrudescence des ulcères gastro-duodénaux 70: 225

-, Kriegszeit, Schwankungen der Manifestation des Ulicus pepticum 75: 147

-, -, warum hat der Magendarmtrakt auf Erlebnisse der - auf Belagerung eigenartig reagiert ? 74: 83

Besnier-Boecksche Krankheit, viscerale Form 65: 36

Bezoare 99: $255(\mathrm{C})$

Bile: v. Fel

Bile ducts: v. Choledocus

Biliary tract: v. Choledocus

Biochimie génétique humaine 100:

135 (L) Bleu de Nil, reaction, moyen d'appré-

ciation de la teneur en graisses des

matières fécales 78: 249 Blinddarm: v. Appendix, Caecum Blood: v. Sanguis Blut: v. Sanguis Blutung: v. Haemorrhagia Bouche: v. Os

Bowel: v. Colon, Intestinum tenue Brill-Symmers, maladie de - à localisation rectale, diagnostic différentiel

avec la recto-côlite 86: 627 Bromsulphalein(B.S.P.) 77:18; 98:129;

99: 182 Brûlures cutanées et ulcère gastro-duo- 
dénal 70: 57

Caecum

82: 189 (A); 85: 237 (A)

-, diseases, non specific inflammatory $83: 34$

-, Volvulus 83: 139

-, fosse iliaque droite, syndromes douloureux chroniques 67: 219

-, Unterbauch, rechten -, chronische Schmerzzustände 67: 169

-, -, Behandlung chronischer Schmerzzustände 67: 186

-, typhlo-appendicite chronique 67: 236

Calcium

-, requirement 91: $338(\mathrm{E})$

-, Stoffwechsel, in Gastroenterologia

90: 192 -, Kalzämie, Hyper-, Verdauungsorgane

99: 195(E) -,'-, Pankreaskarzinom 96: 197

Calculs biliaires: v. Choledocus, Vesica

fellea Canal hépatique, chirurgie du - 99:

401 (L)

278

Index re $\pi$ im ad vol. 64-100

Cancer: v. Carcinoma Carcinoma (v. Neoplasmata) Cancer gastro-intestinal et perte de la spécificité organique 98: 239 (E) digestif, predisposition et anémie per-nicieuse (espagnol) 86: 511

et lesions précancéreuses de Tappa-reil digestif 86: 329

Carcinoidosis, diagnosis and pathology 86: 451

-, gastrointestinal association with apparently congenital malformations 86: 73

Carcinome ( $5^{\circ}$ confer, pathol, géogr.) 85: 126 (L)

- $\quad$ pylorique d'aspect radiologique trom-

peur 64: $286(\mathrm{C})$

-, drüsenbildender Form und Wachs-

tumsgesetze 77: 61 (L) Krebs 69: 118 (L)

- $\quad$ und seine Bekämpfung 75: 62 (L)

Cardia: v. Oesophagus

Carences

alimentaire: ædème de famine 68: 169

- et vitamines 70: $54(\mathrm{~L})$

-, états de - en Belgique pendant l'oc-cupation allemande 1940-1944 72: 285 (L)

protéinique par maladies - 72: $224(\mathrm{R}), 316(\mathrm{R})$

- des gastrectomisés 79: $236(\mathrm{E})$

-, parotidite asymptomatique de - 83: 366 (E)

-, alterations du foie et du pancreas d'origine - au cours de fistules gastro-côliques expérimentales 94: 301

Catalase fécale, procédé clinique d'appreciation quantitative 86: 623

Cellulase, gastro-intestinal -, activity 93: 69

Cellulite et traitement 99: 75 (L)

Chemotherapeutikum, neues Darm- in Dickdarmentzündung 78: 34 (C)

Chirurgia

Chirurgie abdominale d'urgence, strategic et tactique 84: 141 (L)

- $\quad$ digestive, revue des idées 70: 29 (R)

-, experience with second look pro

gramme 100: $263(\mathrm{~T})$

opératoire gastro-entérologique, em.

ploi des ondes électromagnétiques

64: 226

-, Eingriífe abdomineller Risiko bei alien Patienten 84: 275 -, progress in - 100: 132 (L)

Chlorpromazine

- , jaundice due to - 92: 135

,- influence sur les fonctions biliaires

87: 181 -, dilatation of sphincter Oddi by -

92: 42 Cholecystitis: v. Vesica fellea 
Choledocus (v. Fel, Vesica fellea)

64: 56 (R); 65: 119, 298 (A), 378 (A); 66: 49 (A), 108 (A), 245 (A), 355 (A); 67: 98 (A), 111 (L), 159 (A), 274 (A); $68: 97$ (A), 221 (A); $69: 325$ (A); $70: 216$

(A); 71: 363 (A), 365 (A); 72: 411 (A); 75: 376 (A), 386 (A); 78: 69 (L); 80: 387 (A); 81: 353 (A); 82: 246 (A); $83: 357$ (A), 374 (A); $85: 352$ (A); $86: 210$

(A); 87: 121 (A); 88: 335; 89: 375 (A), 383 (A); 93: 409 (A); 94: 54 (A), 401 (A); 95: 134 (A); 99: 136 (A) -, Carcinoma 81: 357 (A); $83: 373$ (A);

89: 379 (A) -, cancer du - et papillome 93: 180 -, chirurgie 81: 358 (A); 89: 381 (A) -, - drainage transpapillaire 88: 373 -, -, quel procédé opératoire choísir pour le rétablissement de la conti-nuité bilio-digestive détruite par fibrose complete des voies - 95: $126-,-$ nerve operations on the tonus of the bile ducts 85: 281

chlorpromazine, influence sur les fonctions biliaires 87: 181

Cholangitis, Pathogenese, Klinik und Therapie 83: 76 (L)

,-- , klinische Bedeutung und Diagno-

stik 87: 135 -, - nach Anastomosen 88: 364

Index rerum ad vol. 64-100

279

Choledocus

,,-- primary stenosing, its relation to carcinoma $88: 305$

-, cyste, congenital idiopathic 92: 251

-, diagnosis and BSP of impaired potency of extrahepatic bile ducts with Rose Bengal 99: 182

-, Endoskopie der Gallengänge 88: 367

-, Erscheinungszeit von intravenös ap-pliziertem Bromsulfalein in der Le-bergalle als Funktionsprüfung des -82: 99

-, evacuation vésiculaịre avec visualisation du cholédoque 82: 107

Gallenwege, Erkrankungen 79: 317 (L); 87: 248, 284 (L)

-, pathologische Anatomie 95: 293 cytologische Untersuchungen des Duodenalsaftes bei Entzündung der -99: 343

lithiase de la voie biliaire principale 89: 149 (L); 94: 330 (L) -, Choledocholithiase, Tomatenschale

als Ursache - 95: 382 -, Steinrezidiv und Gallenwegsana-

stomosen 88: 68 (L) -, désobstruction médicamenteuse 89:

132 (T); 91: 214 (T); 93: 408 (T) Röntgen

Cholangiography, oral or intravenous ? 80: 301 (E); 88: 270

- $\quad$ laparoscopique et indication opéra-

toire $82: 88 ; 88: 275$

-, operative 70: 223 (L); 83: 137 (L) Examen radiologique de la voie biliaire

principale par voie orale $88: 323$ Radiological appearances of biliary tree

and effects of various drugs $83: 2-,-$ investigation of the biliary tract $83: 7$

- $\quad$ - Diagnostik der Gallenwege mit

«Biligrafin» 84: 30 Röntgenologische Darstellung der Papilla Vateri 98: 292

Untersuchungen am terminalen Cho-ledochus bei idiopathischer Choledo-chusdilatation 98: 1

sphincter Oddi 73: 302 (A)

-, -, electric biopotentials 100: 182 -, dilatation by chlorpromazine 92: 42 -, pathologie chirurgicale 89: 204 -, sténose -, pathologie et thérapeutique

87: 268 Papillite sténosante dans la pathologie

biliaire 88: 297 Tubage minute en pathologie cholédo-

cienne 88: 289, 343 -, Therapia 64: $57(\mathrm{~A}) ; 65: 123(\mathrm{~A}) ; 68$ :

223 (A); 69: 327 (A); 71: 366 (A); 73:

299 (A); 76: 34 (A)

Fortschritte in Behandlung komplet-ten Gallenverschlusses mittels Duo-denalsondierungen 65: 162

-, various drugs effects on the radio-

logical appearances of biliary tree 83: 2 -, -, Cholesterinstoffwechsels, Problem 77: 87

Cholestyramin, treatment of pruritus of obstructive jaundice with-100: 62 (T)

Cirrhosis hcpatis (v. Hepar)

75: 268 (A); 79: 244 (A); 81: 342 (A); 83: 319 (A); 84: 205 (A), 262 (A); 85: 358 (A); $86: 809$ (A); 89: 274 (A); $92: 195$ (A), 384 (A); $93: 184$ (A); $98: 126$ (L) alcoolique et nutritionnelle $94: 130(\mathrm{~L})$

-, de Thépatite à la-96: 63 (L)

-, biliäre Leber - 72: 96; 86: 65 -, ceruloplasmin im Serum der -

100: 209 -, -, cholostatic primary - 91: 135 -, classification des - 86: 800 (E) -, électrophorèse 83: 284 -, etiology of liver - 95: 352

alcooliques, fièvre avec ascite 80: 147 -, haemorrhage in - 96: 1

Hanot, maladie de - 86: 308

-, potential upper gastrointestinal bleeding lesions in - 94: 86 -, infantile 86: 406 
98: 393 (L)

-, musculature striée des - 95: 136 (L)

280

Index rerum ad vol. 64-100

\section{Cirrhosis hepatis}

und Narbenleber 82: 250 (L); 88: 67 (L)

Östrogen-Effekt auf dem Plasma-Cortisol bei - 96: 166

Pepsinogen-Serum bei Kranken mit -91: 71

pigmentaire 67: 280 (L) role de la thrombopénie dans hémo-pathies - 99: 402 (L) portal circulation in - 81: 84 telangiektatica bei Morbus Osier S3: 157 Therapia 75: 366 (A); 85: 348 (T) -, cortico-steroids induced biochemical changes in xanthomatous biliary - 89: 343 -, delta-cortisone dans traitement des ascitiques éthyliques 94: $325(\mathrm{~T})$

- , result of strict low sodium diet 93 :

$56(\mathrm{~T})$-, traitement diététique de $-87: 406$

(T); 89: 368 (T) -, Hydrolysatleber-Behandlung der

chronischen Hepatitis und -

81: 300 - perfusion d'ascite, traitement - al-

coolique par - 75: $110(\mathrm{~T})$-, prednisone long-term treatment of

98: $241(\mathrm{~T})$

-, regimes hyperprotidiques désodés, eífets métaboliques dans le traitement des - 84: $261(\mathrm{~T})$

-, spirolactones dans le traitement -97: 205 (E)

-, testosterone dans - atrophique asci-tique 86: $803(\mathrm{~T})$

-, tolbutamide in - 98: $181(\mathrm{~T})$, -, vitamine $\mathrm{B} 12$ à doses massives dans le traitement des - 82: $365(\mathrm{~T})$

varices, esophageal, secondary to

portal - 80: 257

water, diuretic response to - in Laen-

nec's cirrhosis 93: $407(\mathrm{~T})$

Chromatographie d'absorption sur papier, modifications dues aux alcools, des bilirubines 86: 439

Citricämie, Hyper - bei allergischen

Krankheiten 69: 204 (R) Coeliac disease 86: 453 Cæur: v. Cor

Colitis ulcerosa

68: 88 (A); 72: 405 (A); 74: 189 (L); 75: 54 (A), 116 (A); 80: 310 (A); 81: 127 (L); 83: 249 (A); 86: 693 (C), 747 (C); $94: 331$ (L); $98: 245$ (A); $100: 267$ (A) , Arthritis der - 99: 374

$$
\text { et cancer 92: } 233
$$

-, conception actuelle 93: 322 (E) -, coprologique, syndrome 86: 618

en Suisse, données numériques 84: 324

-, acuité de la poussée evolutive initiale

86: 608

grave, anatomie pathologique 86: 632

-, Kolocytogramm 86: 667 fuso-spirochätosa, im deutschen Kon-

zentrationslager 72: 35

-, toxisches Megakolon 94: 29

in einer Vagina artificialis 98: $113(\mathrm{C})$

Recto-côlite ulcéreuse, diagnostic diffé-

rentiel avec maladie de Brill-Symmers rectale 86: 627

muco-hémorragique, lesions histolo-giques 86: 656

hémorragique, poussée grave. Deux cas de «métastase morbide» avec col-lapsus mortel 86: 629 (C)

Ulcerative colitis -, biopsy, rectal 86: 650 -, chronic, etiology, clinic and treatment 86: 760 -, -, European incidence 86: 626 -, exfoliative cytology 86: 338 -, diagnosis 86: 668 -, emotional factors 86: 709 -, familial and ileitis 89: 65 -, life history 86: 549 -, liver function 86: 658 -, mucinases $76: 346$-, natural history 86: 582 -, pathology 86: 666 -, prognosis 88: 13 -, psychosomatic aspects 86: 591

Index rerum ad vol. 64-100

281

Colitis ulceroßa

-, pyoderma in - 99: 311 
-, segmental 92: 157

-, spontaneous remission during jaundice due to virus hepatitis 87: 72 (C)

-, sodium and potassium absorption and excretion before and after colectomy 86: 724

-, Therapia 86: 693, 714

,-- and anti-allergic - 91: 353

-, - antibiotiques-cortisone, et administration par voie rectale de 1'hy-drocortisone 86: 697

-, -, chirurgicalis 86: 718, 754

- $\quad$ - - , indications - chirurgicales et

electro-coagulation pré-frontale 86: 735

- $\quad$ - - chronic - management. Preser-

vation of the rectum $86: 751$

par citrine $72: 372$

274 (L)

-, conservative $-86: 713 ; 99$;

-, -, - ileostomy, post-operative dys

function 86: 752

cortisone in $-81: 86 ; 86: 689$

- with local hydrocortisone hemisuc-

cinate sodium 88: $130(\mathrm{~T})$

kombinierte internistisch-psycho-- 82: 144

traitement par isoniazide associée ou non à la streptomycine et au P.A.S. 94: 258 (T)

Serum-, Chemo- und Vakzine -68: 21

mittels spezifischen Autovakzine-und typspezifischen Enterokokken-Serumtherapie - 68: 36

complications and problems 86: 674

post-operative dysfunction of the ileostomy and its prevention 86: 752

$131(\mathrm{~T})$

radiothérapie diencéphalique 97 :

- $\quad$ mit Salazopyrin 66: 312; 100: 33

-, long-term survival after colectomy

96: 335 (T) Collagénoses 89: 390 (L)

Colon (v. Caecum, Colitis ulcerosa) 66: 242 (A); 67: 269 (A); 68: 92 (A) 69: 214 (A); 70: 102 (A); $71: 245$ (A) $72: 409$ (A); $73: 315$ (A), 374 (A) 75: 51 (A),

116 (A), 125 (A); 82: 190 (A); 83: 253 (A); 84: 140 (A); 85: 237 (A); 86: 125 (A); 87: 117 (A); $89: 142$ (A); $92: 67$ (A); $94: 128$ (L), 133 (L), 264 (A); $98: 254$

(A); 99: 272 (A); 100: 266 (A)

-, Гangle splénique, syndrome de -85: 233 (E)

-, ascending, non-specific inflammatory diseases 83: 34

-, Bacteriologia

Darmbakteriologie, klinische - 82: 252 (L)

Darmflora, Biologie und klinische Be-deutung 68: 5

- und Ernährung 64: 60 (L)

Bakterienflora im oberen Teil des Ver-dauungstraktes und Magensäureaus-scheidung 94: 315

-, Carcinoma 83: 250 (A); 84: 141 (L)

-, -, cancers multiples du gros intestin 89: 180

-, -, Geschwülste, bösartigen - des Dick-darms und Mastdarms 88: 69 (L)

-, -, Dickdarm, Operationswahl bei prä-kanzerösen Erkrankungen des - 86: 372

-, -, precancerous lesions of - and rectum 86: 336

- Intestinaltumoren, Rezidiv nach

Operationen 92: 262

-, origin of chest pain 90: 15

-, chirurgia 94: $333(\mathrm{~L})$

-, -, Kolektomie mit Ileostomie oder mit Anastomose 86: 741

-, -, ileostomy, follow-up of 117 patients 86: 720

-, -, Fistel, Dünndarmkolon - mehr-fache einzeitige Resektion 79: 106

Colitis (v. Colitis ulcerosa) 79:166 (A); 82: 187 (A)

-, right sided 86: 615

hémorragies 83: 252 (A)

-, Crohn's disease 92: 168

282 Index rerum ad vol. 64-100

Colon 
- , Diverticulosis

-, -, klinischer Bedeutung 87: 149 -, - du colon ilio-pelvien 69: 120 (L) -, Diverticulitis coli, stenosierenden, pa-thologisch-anatomische und klini-sche

Erfahrungen und Therapie 84: 315 -, -, Milk in - 100: 61 (T)

- $\quad$ perforativa, eítrige Peritonitis 87: 85

-, Komplikationen des Sigmoïd -

91: 325 -, Dolicho-megacolon 72: 403 (A); 75: 179 (A); 79: 165 (A)

- $\quad$ temporaire d'origine infectieuse

74: $94(\mathrm{E})$

Hirschsprung's disease 76: 339 Megakolon und Megazystis 64: 173 (L)

congenital et idiopathique 99: 264 (E)

transverse d'origine mécanique et occlusion médio-gastrique 67: 55 (C)

-, malformations 65: 356

- $\quad$ Neoplasmata (v. Carcinoma) 75: 170

(A); 79: 178 (A); 83: 253 (A)

,,-- argentaffinoma of the sigmoid 89: 47; 100: 111

-, -, Pneumatosis cystoides des Dickdarms 92: 224; 98 : 163 (C); 100: 111

-, -, tumeurs inflammatoires non spéci-fiques du colon droit 100: 271 (L)

98: 251 (A)

Polyposis 79: $176(\mathrm{~A}) ; 83: 251(\mathrm{~A})$;

-, histological structure in Peutz syn-

drom 97: 119(C) - solitaire du colon descendant 65: 273 (C)

colique disséminée 73: 362

coli, osteomas, cutaneous fibromas and epidermoids 87: 393

adenomatous, as precancerous lesions 86: 370

Ptose isolée de l'angle colique droit par défaut d'accolement 89: 197 (C) Kolonsreiz 96: 120 Stenose des Dickdarms nach Lamin-ektomie 92: 239 -, Therapia

68: 92 (A)

-, -, Darmchemotherapeutikum, neues - bei Behandlung einiger Dick-darmentziindungen 78: 34

-, - Koli-Implantationstherapie zur Normalisierung bakterieller Dick-darmflora. Biologische Grundlagen individueller - 64: 304

-, - Yatrenbehandlung bei Darmkrank-heiten 64: 93

-, Verdoppelung und terminales Ileum 84: 148

-, volvulus aigu du colon droit 76:380 (L)

Coma, prolonged - after small doses of

Insulin 74: 65 Conferences: v. Societates Congrès: v. Societates

Constipation (v. Colon, Feces)

89: 387 (L)

68: 309 (A); 75: 180 (A); 76: 254 (L);

-, traitements $92: 179$ (E) -, chronic, usually a wrong diagnosis 72: 383

provocans (virginum) 77: 165

-, Steuerung der muskulären Darmfunk-tion von der Psyche her 80: 31

Coprologia: v. Feces

Cor

congestive failure in liver 86: 436 coronaire, accident - et affection su-périeure abdomen 90: 1 -, alimentation, cholesterol et açtivité physique 99 : 59 (E)

- $\quad$ sclerosis, relation to peptic ulcer

85: 201; 89: 292, 301

kardiovaskuläre Komplikationen bei regionärer Enteritis 86: 443

changes in postgastrectomy syndrome 89: 315

and gastroduodenal disease, adrenalin discharges in - 73: 219

Herz und Magen, Beziehungen zwi-schen - 81: 177 (E)

Coronaires: v. Cor

Cortisol (v. A.C.T.H.) -, new -, Dexametasone and Triamci-nolone, effect on the shay rate 97: 75

Index rerum ad vol. 64-100 283

Cortisol

-, influence upon the course of obstructive jaundice 82: 193

,-- dans la pancréatite aiguë 84: $73(\mathrm{~T})$; 94: $52(\mathrm{~T}) ; 97: 51(\mathrm{~T})$

-, - etulcèregastro-duodénal92:124(E)

-, Plasma bei Leberzirrhosen, östrogen-Effekt auf - 96: 166

-, ulcerogenic effect 98: 100

Cortisone 
gel à base de - dans traitement des gastrites ædémateuses et hypertro-phiques 88: $262(\mathrm{~T})$

in ulcerative colitis 81: 86

-, delta -, traitement des cirrhoses asci-tiques 94: $325(\mathrm{~T})$-, traitement hepatites ictérigènes 92:

383 (T) hydro -, glucose tolerance test sensitised by - 99: 113 -, local hydrocortisone hemisuccinate

sodium in treatment of ulcerative

colitis 88: $130(\mathrm{~T})$-, par voie rectale dans la côlite ulcé-

reuse 86: 697 Cortico-steroids in xanthomatous biliary cirrhosis, induced biochemical changes 89: 343 - influence upon the number of cells

in the Langerhans Islands 94: 295 -, Wirkung bei idiopathischer Steato-

rrhoe 65: 280 (C) Dexamethason, Behandlung der Hepatitis epidemica 97: 103 Prednisone, long-term treatment of cirrhosis of the liver 98: 241 (T) traitement précoce des hepatites ai-guës $86: 46$

Croissance, stimulants artificiels de - 90: 121 (E)

Cutis

-, cutaneous fibromas, osteomas, epider-moids and polyposis coli 87: 393

-, dermatologische Sicht zur Porphy-rieproblematik 97: 404

-, élastorrhexie, hémorragies gastro-duodénales et - 96: 60 (E)

-, pseudoxanthoma elasticum, haemat-

emesis due to - 89: 345 -, pyoderma in ulcerative colitis

99: 311

Cytologie

-, Cytogramm, colo- bei Colica mucosa und Colitis ulcerosa 86: 338, 667

-, cytologie examination of gastric content in the diagnosis of gastric cancer 86: 378

-, cytology, exfoliative, atlas 82: 63 (L)

-, Zytodiagnostik, Atlas der gastro-enterologischen - 87: 284 (L)

Dentes

-, carie -, addition de fluor aux aliments 76: 297 (E) -, -, EntwicklungsbedeutungderKriegs-

ernährung 76: 252 (L) -, pyorrhée 75: 60 (L)

Decholin, interferierende Wirkung auf der Farbstoffelimination durch die Galle 96: 171

Dénutrition: v. Nutritio

Dermatologia: v. Cutis

Diabetes mellitus

64: $146(\mathrm{R}) ; 65: 125$ (R), 192 (R), 304 (R); 66: 111 (A); 68:100 (A), 331 (A); 69: 330 (A); 70: 219 (A), 347 (A); $71: 369$ (A); $82: 366$ (A)

- $\quad$ infantile et juvenile 84: 271

-, retouches au tableau classique du -79: $318(\mathrm{~L})$

- $\quad$ traumatique 64: $150(\mathrm{R})$

-, praktische Diätküche 68: 112 (L) -, Elektrolythaushalt bei - 90: 182 -, aktuelle Fragen 88: 70 (L)

- und Fruktose-Stoffwechsel: reine

Fruktosurie bei Geschwistern 64: 334

-, gallbladder disease and chronic relapsing pancreatitis 79: 282

- und Hepatitis 75: 273

-, Kalium- und Kohlehydratsstoffwech-sel bei - 76: 276

284

Index rerum ad vol. 64-100

Diabetes mellitus

-, chronic venous congestion and liver

70: 338 - and parotid gland swelling 68: 139 -, therapia 64: 148 (R) -, angiopathia 82: 251 (L) -, Diabétique, foie - 79: 317 (L) -, Zucker, im Harn,

Differenzierung der

verschiedenen - 87: 145 -, -kranker, Bilanzstörungen im Ami-

nosäurenhaushalt $86: 16$

Diabetes renalen, Einfluß von Lactofla-vin über - 73: 17

Diaeta

-, alimentaire, facteur - dans traitement

insuffisances hépatiques 76: 199 -, Diät, kombinierte Purgatio partialis

et generalis und subaquale Darmbä-

der 65: 258 -, diätetischeBehandlung derNahrungs-

mittelallergien 68: 112 (L) -, diet, high-fat - in cases of fatty liver

85: 58 (T) -, Diététique 67: 104 (A), 162 (A); 68: 
105 (A), 326 (A); 69: 336 (A) -, - und Ernährungslehre 76: 318 (L) -, - clinique de Гadulte 78: 70 (L) -, - du médecin praticien 88: 380 (L) -, - precis de - de l'adulte 99: 342 (L) -, - positive en gastro-entérologie 90:

124 (T) -, - traitement - de la cirrhose hépati-

que 87: $406(\mathrm{~T})$-, regime déchloruré, Гemploi des ré-

sines va-t-il remplacer le -? 78:

257 (E) -, - hyperprotéiné, est-il utile dans le traitement de Гhépatite aiguë ? 76: 32(E) -, - désodé - dans le traitement des cir-rhoses du foie: etude des effets métaboliques 84: 261 (T) -, Kuren, Obst- und Obstsaftkuren bei

Kranken und Gesunden 67: 111 (L) -, Diagnose-Differential innerer Krank-

heiten 96: 68 (L)

Diaphragma (v. Hernia hiatalis)

66: 43 (A); 68: 74 (A); 70: 49 (A);

71: $126(\mathrm{~A}) ; 80: 183$ (A); 81: 61 (A);

88: 264 (A) -, abcess, subphrenic 85: 240 (L) -, Zwerchfell, Indikation des Konsti-

tutions 90: 84

Dickdarm: v. Colon

Diuretic response to water in liver disease. I. Acute hepatitis. II. Laennec's cirrhosis 93: 408 (T)

Digestio (v. Gastroenterologia)

-, Digestive diseases, chemistry 97:56(L)

- $\quad$ tract, physiology 99: 276 (L)

-, affections - et accidents coronaires

90: 1 -, appareil, precis des maladies de -81 :

127(L) -, -, et foie 84: $380(\mathrm{~L})$-, maladies - et alimentation de guerre

68: 247 -, pathologie - leçons cliniques (espa-

gnol) 85: 363 (L) -, -, intérêt des mucoprotéines en - 98:

307 (E) -, radiologie -, evolution des idées

69: 71 (R) -, syndromes et pathologie neuro-hor-

monale 70: 223 (L) -, -, viscéro - et leurs traitements

79: 255 (L) Verdauungsapparates, Pharmakologie

des - 66: $213(\mathrm{R})$

krankheiten, Lehrbuch 77: 191 (L); 81: 363 (L); 85: 63 (L)

- und Stoffwechselkrankheiten 77:

$191(\mathrm{~L})$

Organe, Schweizer Heilbäder und Krankheiten der - 67: 68 (R)

-, Erkrankungen, Behandlung

65: $128(\mathrm{~L})$

Divcrticula (v. Duodenum, Colon) -, de Гestomac à Гintestin grêle 89: 227 -, quand faut-il les opérer les -? 99: 336 (E) perforativa $89: 257$

Index rerum ad vol. 64-100

285

Diverticula

des Magen-Darmtraktes, chirurgische Behandlung 89: 257

Grasersche - 85: 33

Douleurs viscérales, irradiation, essai

d'objectivation 77: 380 (E) Duodenalsondierung: v. Vesica fellea

Duodenum (v. Intestinum tenue, Ulcus pepticum)

68: 84 (A); 71: 240 (A); 74: 365 (A); 82: 184 (A); 84: 138 (A); 85: 235 (A); 92: 60 (A); $98: 185$ (A)

Karzinom 92: 207

neurinoma developed around metal foreign bodies $98: 354$ (C)

gutartige Tumoren 72: 60

proteolytische Fermente 82: 1

Duodenal, mucosographie -, pour diagnostic duodénite 93: 44

-, - saft,Leukozytenbefundim-96:153 -, - schleimhaut, Retroprolaps der -80: 208 (C)

sondenflüssigkeit und Typhusdauer-ausscheider, bakteriologische Unter-suchung 94: 1

stases, étiologie et traitement 87: 285, 355; 88: 31, 10, 214, 232

stenose, arteriomesenteriale 89: 170

syndrom und intestinale Amöbiasis 91: 42

-, Bulbus, Erosionen 97: 188 Duodénites, frequence et traitement 
77: 99 -, Diverticula 68: 85 (A); 76: 374 (A);

75: 48 (A); 79: 163 (A) -, -, as the cause of chronic pancreatitis 80: 203

et pancreas 89: $389(\mathrm{~L})$

-, Dyspepsie, bioptische Befunde des -96: 137

Dysenteries: v. Parasiti 71: 251 (A)

Eau: v. Aqua

Einfrieren des Magens zur Behandlung

rezidivierenden Zwölffingerdarmge-

schwürs 100: 65

Eisen: v. Ferrum Eiweiß: v. Protein

Electro-coagulation et cancer du rectum 85: 117 (T)

pré-frontale dans la recto-côlite hé-

morragique 86: 735

Electrolytes

and aldosteron bei Leberkrankheiten 90: 216

changes, effect on intestinal motility 85: 142

bei Diabetes mellitus 90: 182

des selles 96: 217, 223, 312, 326

und Wasseraushalt 90: 133, 149, 204; 94: 130 (L)

Electrophoresis

-, zur Analyse der Magensaft- 85: 10

dans cirrhose hépatique $83: 284$

-, fractionation of human gastric juice

and saliva 84: 99 -, immunoelektrophoretische Studien

an Organextrakten des Magens 98: 30 -, method for characterization of juices

of gastro-intestinal tract 97: 31

Emetine, 2-déhydro-, amæbicide syn-thétique susceptible de remplacer $\Gamma-97: 136(\mathrm{~T})$

Endocrines (v. Parathyreoidea, Sex, Surrenal, Thyreoïdea) 66: 50 (A), 358 (A); 67: 102 (A), 161 (A); 96: 68 (L)

Entérites: v. Ileum, Intestinum tenue

Enzyme

- $\quad$ inhibitors, in therapy of gastro-duo-

denal ulcer 86: 485; 100: 63 (T)

-, Resorption durch das Peritoneum

97: 8 -, zuckerspaltende chronische Durch-

fälle, verursacht durch einen Mangel

an - 97: 349 -, spezifischer optischer Fermenttest,

in Parenchymschädigungen der Leber

87: 172

Eosinophilia, Ernährungsbedingte

77: 373

286 Index rerum ad vol. $64-100$

Eosinophilia

et parasitoses 79: $44(\mathrm{E})$

Ephedrins, "Wirkung auf Pepsinogen-Aktivität des Plasmas 94: 182

Epidemiology and digestive disease 89: 363 (E)

Ernährung: v. Alimentatio, Nutritio

Esophagus: v. Oesophagus

Estomac: v. Stomachus

Ether, treatment of retained postoperative biliary stones 93: $408(\mathrm{~T})$

Feces (v. Colon, Constipation, Intesti-num tenue)

Coprologie clinique 75: 389 (L)

Diagnostica coprologica 98: 320 (L)

Fel (v. Icterus)

bile acids, conjugation after partial hepatectomy 99: 327

Gallenfarbstoffe, Biologie der - 98: 192 (L)

Felty Syndrom 75: 129 Ferments: v. Enzym

Ferrum

Eisenmangel, Dumpingsyndrom und larvierter - 82: 14 
Eisenstoffwechsel 96: 66 (L)

Iron absorption and storage in sidero-penia 93: 113

Fett: v. Lipid

Fettsucht: v. Obesitas

Fistules gastro-coliques expérimentales, alterations carentielles du foie et du pancreas 94: 301

Fluor, addition - aux aliments peut-elle éviter la carie dentaire ? 76: 297 (E)

Foie: v. Hepar

Folic acid, deficiency, effect on gastrointestinal tract 89: 347

Fosse iliaque droite, syndromes douloureux (v. Caecum) 71: 379 (L)

Freezing, gastric - achieving physiological gastrectomy 99: $62(\mathrm{~T})$

Frei Test, positive in Morbus Crohn 99: 368

Fructose: v. Glucids Fuso-spirochaetosa, Colitis ulcerosa -

72: 35

Galaktose: v. Hepar

Gallbladder: v. Vesica fellea

Galle: v. Fel

Gallenblase: v. Vesica fellea

Gallensteine: v. Choledocus, Vesica

fellea Gallenwege: v. Choledocus Gallstones: v. Choledocus, Vesica fellea

Gastrectomia (v. Stomachus: Chirurgia, Therapia, Ulcus pepticum)

-, afferente Schlingensyndrom nach Bill-roth II, Symptomatologie und Thera-pie 94: 90

- et assurance-vie 86: 119 (E)

-, anemia, postgastrectomy megaloblas-tic - and the loop syndrom 89: 326

-, Beschwerden, Behandlungsergebnisse von Magenresezierten 87: 78

-, blood volume before and after gastrectomy 92: 137

-, cardiovascular changes in postgastrectomy syndrome 89: 315

-, carence protidique des - 79: 236 (E)

-, dehydrogenase activity, relation between rates of secretion and intracel-lular-99: 169

-, douleur-fantôme des gastrectomisés 85: 341 (E)

-, gastroskopisches Bild der - 86: 775

-, Dumping syndrome 75: 168 (E)

-, Durchblutung der Darmmukosa beim - 97: 1

,-- und larvierter Eisenmangel 82: 14

-, - nach Magenresektion, Resorption von Vitamin A 95: 34

-, early postgastrectomy syndrome, etiology 76: 319

-, gastric acid secretion before and after removal of pyloric antrum 89: 286

-, gastric freezing, physiological -99: $62(\mathrm{~T})$

Index rerum ad vol. 64-100

287

\section{Gastrectomia}

- polaire supérieure avec anastomose

continente 98: $390(\mathrm{~T})$-, relations between gastritis, peptic

ulcers and gastric carcinoma in -

95: 341 -, hemorrhage, upper gastro-intestinal,

following - 98: 93 -, Häufigkeit der Hiatushernie nach -

96: 99 -, histamine test, augmented in - 99: 30 -, ileo-jejunal insufficiency following different types of - 81: 104 -, ileus après - 66: 174 -, changes in jejunal function after partial - 95: 1 -, Leberbefunden, Häufigkeit von pa-

thologischen - 96: 158 -, distal, metabolism of carbohydrate in

- 99: 394 -, Einfluß auf die Morphologie der

Schleimhaut des Magen-Darm-Trak-

tes 95: 36 -, Nahrung, Ausnützung der - 91: 76 -, operation for post - syndrom 92: 146 -, le colon peut-il remplacer एestomac ?

78: 64 (E) -, Osteomalazie und hypochrome An-

ämie nach - 66: 182 -, postcibal symptoms after partial - for

peptic ulcer 81: 362 (L) -, Proteolyse, fermentative in magen-

losen Patienten 96: 103 -, pulmonary, ventilation in - patients

99: 54 -, radiologische Aspekte der Antrum er-

haltenden - 83: 330 -, reflux alimentaire dans le duodenum 
89: 192 -, results, late, medical aspects 82: 253;

85: 195 -, Total, physiopathology, symptomatology and medical management

85: 80 -, -, digestion after - 85: $116(\mathrm{E})-,-$, androgenic hormones, protein effect in treatment following - 93: $324(\mathrm{~T})$

- , résultats 93: 193

trace elements in serum of partially -

patients 95: 374

Tuberculosis following partial -

81: 91; 90: 65

-, facteurs pathogéniques de la -

84: 17 Verdauungstrakt, Anpassungsformen nach - 99: 45

Gastroenterologia (v. Digestio)

67: $109(\mathrm{~L}) ; 72: 66(\mathrm{~A}), 79(\mathrm{~L}) ; 75: 61$

(L); 78: 332 (L); 81: $52(\mathrm{~A}), 363$ (L);

82: $50(\mathrm{~A}) ; 83: 128(\mathrm{~A}), 321(\mathrm{~L})$;

84: 74 (A); 85: 51 (A); 86: 54 (A);

87: 59 (A), 126 (L), 268 (A); 88:

67 (L), 202 (A); 89: 55 (A), 390 (L);

90: $55(\mathrm{~A})$; $91126(\mathrm{~A})$; 93: 57 (A);

94: 119 (A); 96: 64 (L); 95: 64 (A);

96: 67 (L); 97: $208(\mathrm{~A}) ; 98: 191(\mathrm{~L})$;

99: 63 (A), $74(\mathrm{~L}) ; 100: 134(\mathrm{~L})(\mathrm{L})$

200 (A) -, consultations journalières 97: 54 (L) -, confrontations, radio-anatomo-clini-

ques $72: 360(\mathrm{~L}) ; 73: 384(\mathrm{~L}) ; 75: 271$

(L); 77: 395 (L); 81: 360 (L) -, diététique positive en - 90: 124 (T) -, formulaire - du praticien 75: 271 (L) -, pharmacologie et - 69: 307 (R) -, psychosomatique et - 99: 277 (L) -, alimentary tract, disorders of the - in

relation to haemopoiesis 79: 322 -, Gastro-entéropathie exsudative

96: 83; 97: 340 -, gastrointestinal juices, electrophore-

tic method for characterization 97: 31 -, -, physiology 99: 276 (L) -, -, Probleme im Kindesalter 90: 301 -, - Symptome beim Lupus erythema-

todes visceralis 95: 155 -, - motility in man. Effect of a new parasympathetic blocking agent 82: 225 -, -, pathologie -, role du système ner-

veux végétatif 72: 420 (L) -, -, troubles - dans Гinsuffisance thy-

roïdienne 74: $225-,-$, primary - tuberculosis $99: 359$

288

Index rerum ad vol. 64-100

\section{$\mathrm{Ga} \beta \mathrm{t}$ 「oeneterologia}

-, Magen-Darmtrakt Reaktion auf Er-lebnisse des Krieges und der Belage-rung 74: 83 -, -, Kanal, pH-Messungen 75: 138 -, -, Krankheiten und Hepatopathie 79: 1 -, -, -, liegt eine Zunahme der - in jet-ziger Zeit vor ? 68: 226

Gastroscopie (v. Stomachus) 64: 61 (L), 63 (L); 65: 290 (A); 72: 216; 73115 (A); 75: 391 (L); 78: 68 (L) 87: 127 (L); $94: 199$ (L); $98: 393$ (L) 99: 77 (L)

-, endoskopischer Beitrag zur Salzsäure-verätzung des Magens 76: 293

- - Diagnostik des Magensarkoms 76: 127

-, Befund bei Anazidität 67: 125

-, Betrachtungen nach Bauchoperatio-nen 66: 298

-, Erfahrungen bei 4500 - mit Rück-sicht auf die Indikationen 82: 300

-, pharmako-radiologische und gastro-skopische Untersuchung des Verdau-ungskanals mit «Teab» 80: 220

Gelée royale, valeur alimentaire et bio-

logique 87: 343 (E) Genetics, human and congenital pyloric

stenosis 97: 50 (E) Geschwür: v. Uḷcus pepticum Geschwulst: v. Carcinoma, Neoplasmata Gesellschaft: v. Societates

Glucids

Fruktose - StoíFwechsels, Pathologie

64: 334

glucose tolerance test sensitised by

hydrocortisone 99: 113

glycémie au cours de la maladie ulcé-

reuse 73: 209

Hypoglycémie 68: 333 (A)

-, dumping syndrome and jejunitis

79: 16 -, causation of abdominal pain in peptic ulcer 86: 535 hydrates de carbone, métabolisme, role du foie 70: 198 (R)

-, -, Stoffwechselstörungen, angeborene 
97: 234 -, - Resorption aus dem sterilen Darm-

kanal 83: 349 -, Polysaccharids, Wirkung auf die pep-

tische Proteolyse 96: 85, 91 -, Zuckerstoffwechsel-Störungen nach Hepatitis epidemica 71: 83

Glutamyl-transpeptidase, gamma- in internal diseases 99: 237

Gluten and antibacterial agents influence on fat absorption in sprue 81: 98

-, toxicity, clinical and biochemical analysis 94: 227; $97: 149$

Glycémie: v. Glucids Goutte: v. Podagra Graisses: v. Lipid Guerre: v. Bellum

Gynergen und Dehydroergotamin, Be-handlung des Ulkus mit - 72: 135 (E)

Haemorrhagia

-, aspirine et - 71: $274(\mathrm{E})$

bleeding, gastrointestinal potential

upper - lesions in cirrhotic patients

94: 86

massive Magen-, Klinik und Therapie

77: 60 (L); 100: 263 (T)

-, - Behandlung der - 84: $173(\mathrm{R})$

-, tödliche, durch das Aneurysma

eines Magengefäßes 96: 383 (C) -, haemorrhage, changes in the

splanchnic circulation after -

81: 72 -, - in cirrhosis of the liver 96: 1 hemorrhage gastro-intestinal, reser-pine induced 96: 395 (T) -, -, following gastrectomy 98: 93 -, -, azotemia 84: 70

(E) hematemesis and melena, special reference to bleeding of unknown origin 85: 97 - due to pseudoxanthoma elasticum

89: 345 hémorragies provoquées par Гaspirine en dehors de Гulcère 96: 69 (L)

Index rerum ad vol. 64-100

289

Haemorrhagia

-, - gastro-duodénales par élastorrhe-

xie 96: 60 (E) -, -, sine materia, manifestations de la

goutte 100: 53

Harnverhaltung, spastischer Ileus in-folge - 77: 348

Haut: v. Cutis

Heart: v. Cor

Heinz bodies during Azulfidine treatment of ulcerative colitis 100: 33

Hefe- und Schimmelpilzallergien mit ga-strointestinaler Reizantwort 89: 153

Hémorroïdes: v. Anus

Hepar (v. Cirrhosis, Fel, Haemorrhagia, Icterus, Porta)

65: 111 (L), 296 (A), 378 (A); 66: 245 (A), 355 (A); 67: 95 (A), 272 (A); 68: 95 (A); 71: 346 (A); 73: 204 (A); 75: 272 (L), 370 (A); $77: 64$ (L), 77 (L); $78: 69$

(L); 79: 315 (A); 82: 64 (L), 245 (A), 251 (L); 83: 320 (A); 85: 361 (A); 86: 809 (A); 87: 119 (A), 126 (L); 89: 151 (L), 372 (A); $92: 390$ (A); $93: 188$ (A); $94:$

329 (A), 332 (L), 394 (A); 97: 215 (L); $98: 134$ (A), 309 (A), 391 (L), 394 (L); 99: 134 (A), 275 (L), 339 (A); 100: 207 (L), 269 (A)

-, abcès du foie 64: 63 (L)

-, Adenome, Leberzell - ohne Zirrhose 99: 1

-, ammoniémie et affections hépatiques 86: 421

-, Bilirubin im Serum, quantitative Untersuchungen über - und Harn 74: 76

-, blood flow, intrinsic factors in regulation of -81: 81

-, Bromsulphalein Ausscheidungskurve, Frage der «normalen» - 95: 18

-, - in der Lebergalle als Funktionsprü-fung des Choledochus 82: 99

-, Funktionsprüfungen, kombinierte -mit besonderer Berücksichtigung der Bromsulphaleinprobe 77: 18

-, Carcinoma 79: 313 (A); 81: 352 (A); 85: 361 (A); 89: 371 (A)

-, -, métastatique 67: 66 (C)

,,-- psammo $-71: 303$

-, cardiac, congestive - failure and cons-trictive pericarditis $86: 436$

-, «cholérétique-morphine», test -97: 137

-, Koma, Physiopathologie 90: 235

-, - hepaticum, therapia 85: $241(\mathrm{R}) ; 86: 395 ; 90: 291$

-, - acide thioctique, biocatalyseur hé-patotrope, son role dans le -89: 131 (T)

-, cuivre, foie, système nerveux 93: 255 (E)

-, Diabète et foie 79: 314 (A)

,-- mellitus, chronic venous congestion of $-70: 338$ 
-, Diagnostic (v. Bromsulfalein, Explo ration fonctionnelle, Galactose, Pone tion-biopsie, Rose Bengale) 64: 53 (R) 65: 121 (A); $68: 166$ (A); 69: 222 (A) 70: 105 (A); 71: 356 (A); 73: 189 (A) 75: 181; 79: 240 (A); 81: 246 (A) 82: 242 (A); 83: 316 (A); 89: 266 (A) 93: 182 (A)

-, foie et appareil digestif 84: 380 (L) Elektrolyte und Aldosteron bei -Kranken 90: 216

étio-pathogénie des affections hépatiques non infectieuses (ital.) 83: 257 (L) exploration fonctionnelle 79: 318 (L); 241 (A)

-, nouveau procédé d'-: mesure du temps de prothrombine 67: 111 (L) -, vereinfachte Alkoholtest als -pruning 74: 341 -, Fermenttest zum Nachweis spezifischer optischer Parenchymschädi-gungen 87: 181 Farbstoffausscheidung, pharmakolo-gische Beeinflussung 96: 176 Hepatikusgabel Syndrom 96: 190 Galaktoseausscheidung, Wirkung des Histamins bei Menschen nach oraler Galaktosebelastung 66: 57 - belastung, Versuche 75: 193 gastro-duodenitis, liver changes in chronic - 93: 385

24 Gast $\Gamma o e n t e r o l o g i a$, Vol. 100, No. 5/6 (1963)

290

Index rerum ad vol. 64-100

\section{Hepar}

-, Magenschleimhaut bei chronischen -

-Kranken 90: 373 -, - Darmkrankheiten und Hepatopa-

thie 79: 1 -, Gefäßveränderungen bei Lebererkran-

kungen 96: 163 -, Gehirn, Auswirkung chronischer Le-

berkranken auf - 90: 259 -, foie et transport des graisses 80: 1 -, hémochromatose, dangers du traite-

ment par saignées 87: 186 -, Hepatitis 69: 227 (A), 321 (A); 70:107 (A); 73: 194 (A); 75: 261 (A); 79: 243 (A), 309 (A); $81: 250$ (A); $82: 244$ (A); $83: 317$

(A); 84: 203 (A); 86: 805 (A); 89: 271 (A); 92: 193 (A)

de $\Gamma$ - à la cirrhose alcoolique 96: 63 (L)

und Diabetes 75: 273 differential diagnosis, value of ACTH administration 87: 23 acute -, diuretic response to water 93: 407 (T)

Duodenalsaft bei Patienten mit -99: 343

epidemica, Dünndarmveränderun-gen bei - 70: 24

-, Blutplasmazellen anhand von Milzpunktaten 71: 97

- $\quad$ klinische Abgrenzung der - von

andern Hepatitisformen 68: 113

14 Todesfälle an - 69: 258 (C)

-, pathogénie, anatomie pathologi-que 71: 62

métabolisme de la thyroxine dans - 87: 129

Gelbsuchtkrankheiten 69: 232 (L)

Galaktose, Tag- und Nachtproben

im Verlauf der -. Prognotische Be-

deutung 94: 171 - infectieuse aiguë, recherches biopsiques $74: 1$

, notions actuelles 80: $238(\mathrm{E})$

-, effect of gamma-globulin 96:264 (T) -, virale, C. R. 4e Conf. Pathol. Géogr.

81: $126(\mathrm{~L})$

-, Inokulations -, Verhütung 76: 9 -, iproniazid administration, hepatic

necrosis by - 98: 273 (C) -, lupoide -, Syndrom 100: 220 -, Ornithose mit hepatitischem Bild

98: 15 -, post - Hyperbilirubinämie 84: 207 -, - Lebererkrankungen 91: 1 -, - syndrome 83: 77 -, epidemica, Zuckerstoffwechselstö-

rungen nach $-71: 83-,-$, séquelles (v. posthepatitic) 71: 4,

295 -, -, Therapy, ACTH-treatment 83:

148, $313(\mathrm{~T})$, -, épidémique, traitement chirurgical

87: $49(\mathrm{~T})$-, épidémique, intervention chirurgi-

cale d'urgence au cours de - 87: 325 -, ictérigènes, traitement par de la

cortisone 92: 383 (T) -, epidemica Behandlung mit Dexa-

methason 97: 103 -, aiguës, diététique actuelle 89:

368 (T) -, ictériques infectieux, faut-il les

pousser à manger beaucoup? 83:

311 (E)

aiguës, traitement précoce par la Prednisone 86: 46

-, regime hyperprotéiné est-ilutile ?

76: 32 (E)

-, Sympathektomie periarterielle bei schwerer - 96: 203

-, Übertragung parenteralen - 77: 329

-, ulcerative colitis, spontaneous remission of - during jaundice due to virus 87: 72

histologic changes of liver in diseases 
of the biliary tract 91:221

role des hydrates de carbone dans

métabolisme - 70: $198(\mathrm{R})$

insuffisance, soi-disant petite, -93 :

405 (E)

nal 83: 100

hépatique et ulcère gastro-duodé-

Leberinsuffisanz, akuter, Klinik 90: 271

Index rerum ad vol. 64-100

291

Hepar

-, Klinik und Therapie 90: 240

-, nouveaux traitements de $\Gamma$ -

75: 59 (L) intra-hépatiques, maladies des voies biliaires - 97: 129 (E) Kindesalter, Leberkrankheiten im -85: 125 (L)

kystique, maladie - 79: 309 (A); 82: 379 (L)

lentikuläres, Hepato-Syndrom im Alter 79: 299

maladies diverses 64: 55 (R); 65: 119 (A); 66: 57 (A); 68: 167 (A), 217 (A) 69: 322 (A); 70: 108 (A); 71: 351 (A) 72: 96 (A); $73: 199$ (A); $75: 370$ (A) 81: 349 (A)

terminaisons nerveuses dans le -79: 220

aspect endoscopique de Гæsophage dans quelques hépatopathies 86: 266 osteitis deformans Paget, position of hepatopathy within etiology of -64: 84

ovarien, syndrome hépato -75: 60 (L)

hépato-pancréato-biliaires, correlations - 83: 243 (E) Leber und Pankreas-Enzymologie 98: 125 (L)

pancreatitis development, role of diseases of liver and biliary tracts in -80: 263

-, secretion au cours certaines affections hépato-biliaires 72: 51 Physiologie 69: 220 (A); 70: 104 (A); 71: 343 (A); $79: 238$ (A); $83: 315$ (A) Physiopathologie 81: 240 (A); 82: 240 (A); 84: 201 (A); 85: 356 (A); 92: 182 (A)

Ponction-biopsie 69: 225 (A); 75: 389 (L); 79: $240($ A)

- recovery from bile peritonitis following needle - 80: 357 -, Befund und Leberfunktionsproben

84: 349 -, confrontation entre les lobes droit et gauche du foie 85: 65

, -, Leber Ergebnisse, Anzeigen, Ge-

fahren 94: 137 , -, Leber und Hepatogramm 77: 163 , -, new method of liver puncture 74:

349 , Proteinkomponenten der geschädig-

ten - 81: 129 , Dysproteinämieentstehung bei Leber-erkrankungen, experimentelle und klinische Untersuchungen 95: 88, Regenerationsfähigkeit,

Beobachtun-gen der menschlichen Leber 85: 250 , -, action de l'acide adénosine-tri-phosphorique sur capacité de -(ital.) 81: 153, rose Bengal, radioiodịnated, uptake and excretion in liver disease 86: 430 , - -Prüfung, Leberdurchblutung mit

Radio - Untersuchungen 91: 303 , Struktur und Funktion 97: 216 (L), Takata-Ara-Reaktion in Diagnose der

Lebererkrankungen 64: $23,-$ signification de la reaction 70 :

88 (R) , temperature of human liver parenchyma 83: 233 , Therapia (v. hepatitis) 64: 57 (A); 65: 123 (A); 68: 219 (A); $69: 324$ (A); $70: 108$ (A); $71: 358$ (A); 79: 310 (A); 92: 191(A) , -, Androgene und anabole Steroide, Leberschädigung durch neue - 96: 263 (T) , -, Chirurgie, 95: 306; 96: 64 (L) ,--, anastomoses bilio-digestives intra-hépatiques 86: 507 - - artère hépatique, interventions sur - dans le traitement des maladies du foie 79: 156; 100: 132 (T) -, exérèses périkystiques dans les kystes hydatiques du foie 80: 139 -, - hepatectomy, conjugation of bile

acids after partial - 99: 327 -, -, Behandlung schwerer blutiger

Leberverletzung 91: 388 -, -, regeneration after partial hepatectomy 100: 75

292

Index rerum ad vol. 64-100

\section{Hepar}

,-- , cholérèse et cholérétiques 92 :

59 (T) -, -, Diaeta 73: 202 (A) -, -, - high-fat - in cases of fatty liver

85: 58 (T) -, -, - insuffisances hépatiques, facteur alimentaire dans le traitement -76: 199 -, -, - regime hyperprotéiné est-il dan-gereux pour les hépatiques ?

88: 259 (E) -, -, Lebertotalextrakten, Behandlung von Leberparenchymkrankheiten 82: 270; 93: 300 -, -, lipotropen Substanzen, Prevention der Lipophanerose durch - 90: 329 -, -, fallacy of low fat diet 100: 132 (T) -, syndromes ulcéro-biliaires 82: 19 -, veine porte et foie 85: 363 (L); $86: 64$ (L)

Hernia hiatalis (v. Diaphragma, Oesophagus) 65: 279 (C); 76: 56 (A); 78: 264 (A); 83: 136 (A); 86: 315, 319; 89: 61 (A); $90: 57$ (A); $91: 132$ (A); $93: 61$ (A); 94: $126(\mathrm{~A})$

- $\quad$ in bronchial asthma 93: 337 ; $94: 351$;

95: 110

-, closing mechanism between oesophagus and stomach 86: 219

-, Diagnose der kleinen - 82: 76 -, radiological 78: 346 diaphragmatica dextra oder Even-tratio 80: 20 -, role du - dans la continence gastro- 
æsophagienne 86: 232 -, extrahiatal 75: 9 gastro-oesophageal junction 99: 277 (L)

nach Magenresektion 96: 99 péristaltisme cesophagien dans le mé-ga-cesophage et la - 86: 151

- $\quad$ postérieure droite du hiatus pleuro-

péritonéal (foramen de Bochdalek)

80: 99 (C)

-, radio-physiologie de Гextrêmité infé-rieure de Гoesophage 86: 242

- , reflux, gastro-cesophageal in - 86: 272 ;

89: 340 -, - and æsßphagitis, treatment 86: 287 -, sphincter, gastro-cesophageal in healthy human beings 86: 135

Hernie 68: 87 (A) Herz: v. Cor

Histamine (v. Stomachus, Ulcus pep-ticum)

-, role in production of peptic ulcer 86: 489

-, stimulated and basal gastric secretion and acidity in duodenal ulcer 96: 351; 97: 39

-, number of parietal cells and rates of gastric secretion before and after subcutaneous injection of large doses 98: 207

-, augmented test -, clinical experience in gastrectomy 99: 30

Histidinbehandlung allergischer Krank-heiten 71: 233 (R)

Histoire de la médecine et du livre medical 99: 274 (L)

Hormones, traitements de Гulcère gas-tro-duodénal par - 69: 31

Hunger diseases during period of famine 1945 71: 327

Hydrargyrum

mercury salts, effects on gastric secretion 99: 105

Mersalyl (Mercurgan), inhibition of gastric secretion of acid with - 86: 381

Hydrates de carbone: v. Diabetes melli-tus, Glucids

Hydroxy-tryptamine

endogen -, pharmakologische Wïr-kung auf Magen und Darm 90: 382

und Erkrankungen der Verdauungs-organe93: 235; 96: 275

Hypoglycemia: v. Glucid

Hypophyse 64: 155 (R) -, Vorderlappen und Magengeschwiir 82: 162

Index rerum ad vol. 64-100

293

Hypophyse

-, pituitary glands influence on gastric secretion 85: 205

Icterus (v. Fel, Choledocus, Hepar, Ve-sica fellea)

69: 339 (L); 75: 189 (A); 85: 357 (A); 89: 369 (A); 99: 401 (L) atypische Dubin-Johnsonsche Syn-drom 94: 201

diagnostic différentiel, regies cliniques 87: 47 (E)-, cholinestérase sérique 79: 111

et thérapeutique des - 78: $68(\mathrm{~L})$

par retention 83: $318(\mathrm{~A})$; 87: $62(\mathrm{~L})$

-, thérapeutique chirurgicale

94: 334 (L) neonatorum 98: 395 (L) Differentialdiagnose als Grundlage für chirurgische Eingriffe 95: 315

zwischen Verschluß und Leberzell -

80: $380(\mathrm{E})$

Physiologie und Pathologie des Bili-rubinstoffwechsels als Grundlagen der 77: $61(\mathrm{~L})$

obstructive jaundice, influence of cor-ticoids 82: 193

prolonged jaundice due to chlorpro-mazine 92: 135 -, treatment of pruritus with chole-

styramine 100: $62(\mathrm{~T})$

Ileum (v. Intestinum)

84: 139 (A); 85: 235 (A); 98: 187 (A) diverticule Meckel 76: 374 (A) Ileitis regionalis 74: 378 (L); 80: 303 (A); $92: 64$ (A); $95: 258$ mit symptomatischer Sprue 81: 1

kardiovaskuläre Komplikationen bei - 86: 443

bei einem weiblichen Zwillingspaar 89: 75

-, life history 89: 352

-, Röntgenologie der - 95: 268

-, Chirurgie der - 95: 281

-, pathologische Anatomie 95: 220

Morbus Crohn, positiver Frei Test in -

99: 368

-, -, Ätiologie der - 94: 47 (E) 
-, Crohn's disease of colon 92: 168

à debut cæcal 96: $235(\mathrm{C})$

chez le nourrisson 95: 48 Ileitis follicularis 98: 19

and ulcerative colitis, familial 89: 65 -. Ileocolitis granulomatous lesions in -97: 191

Infections, gastrointestinal in animals, relationship to similar infections in man 83: 28

Intestin grêle: $v$. Intestinum tenue

- gros: v. Colon

Intestinum (v. Colon, Duodenum, Ileum,

Jejunum, Rectum)

65: 294 (A), 376 (A); 66: 117 (L); 67:

86 (A), 156 (A); 68: 93 (A); 79: 182

(A); 80: $383(\mathrm{~A}) ; 83: 256(\mathrm{~L}) ; 85: 234$

(A); 90: 124 (A) -, anse borgne, syndrome 94: 116 (E) -, Autointoxikation, als Ursache schwe-

ren chronischen Blepharokonjonkti-

vitis 67: 147 -, biopsy 100: $272(\mathrm{~L})$-, troubles circulatoires 79: $176(\mathrm{~A})$-, diagnostic 65: 113 (A); 66: $104(\mathrm{~A})$;

68: 90 (A), 304 (A); 94: 261 (A)

diarrhées du nourrisson, traitement

des - 85: $63(\mathrm{~L})$

,-- classification according to functional pathology 90: 358 -, - graves par antibiotiques 81:

238 (E) -, Durchfälle, chronische, verursacht

durch einen Mangel an zuckerspal-

tenden Enzymen 97: 349 -, duplication intestinale, cure opéra-

toire d'une - 87: 218 (C) -, Entéropathies et déperdition de pro-

téines 98: 65 -, --, aspects chirurgicaux des - 98: 76 -, Infections 65: 115 (A); 66: 354 (A);

68: 310 (A); 75: 39 (A); 79: 171 (A) -, inflammation, examens morphologi-

ques concernant Faction des remèdes

sécrétoires sur - 65: 63

294

Index rerum ad vol. 64-100

Intestinum

-, Meteorismus. Röntgenologische Sym-ptome und Verwertung bei Diagno-stizierung einigę $\Gamma$ Darmerkrankungen 66: 162

-, Infusionsödem der Darmwand 90: 231

-, operations, mageloblastic anaemia following - 79: 338

-, Pathologie 94: 261 (A)

-, Physiologie 66: 103 (A); 75: 17 (A); 76: 363 (A); 79: 158 (A); 80: 306 (A); 94: 259 (A)

-, pneumatosis cystoïdes - 65: 193, 313; 92: 212

-, Polypose gastrointestinale mit Mela-nose der Lippen und Mundschleim-haut 84: 341

-, pyxigraphie 87: 349

-, Radiologie 68: 90; 76: 366 (A); 80: 309 (A)

-, resections, regenerative metabolism and utilisation of food in extensive gastro-intestinal - 86: 543

-, Therapia 65: 117 (A); 66: 107 (A); 68: 312 (A); 69: 216 (A); 74: 296 (A); 75: 38 (A)

-, Tuberculose 78: 72 (L); 98: 253 (A)

,-- , chirurgie et radiologie 92: 16

,-- ulcéreuse iléo-caecale. Indications actuelles du traitement chirurgical 80: 129

,,-- traitements nouveaux 73: 372 (E)

-, Verdoppelung von terminalen Colon 84: 148

Inseln, Langerhansschen -, Hyperfunk-tion durch chronische Bauchspeichel-drüsenentzündung 66: 72

Insulin 98: $54(\mathrm{E})$

-, prolonged coma after small doses of -74: 65

Intestinum tenue (v. Duodenum, Ileum, Jejunum, Parasiti, Steatorrhea) 66: 46 (A), 240; 67: 265 (A); 69: 212 (A); 70: 1 (O), 101 (A); $71: 242 ; 72.357 ; 73$ :

309; 74: 372; 75: 39, 47; 76378 (A); 83: 249 (A); 84: 380 (L); 86

124 (A); 88: 138 (A); 89: 388 (L); 92: 66 (A); 95: 68 (A); 99: 269 (A); 100: 265 (A) -, absorption, site of fat - in the rat 85: 160

-, some aspects of the cellular phase of fat $-85: 146$

laboratory diagnosis $94: 20$

der Kohlenhydrate auf dem steri-len Darmkanal des Kaninchens 83: 349

of isil-labelled fats. Effect of experimental cholecystectomy on -99: 301

-, intestinal 99: $276(\mathrm{~L})$ 
-, nouvel agent pour faciliter - des graisses 75: 38 (E)

Biopsy 100: 272 (L)

-, neues Instrument 96: 143

Entzündungen, Tierexperimentelle

Untersuchungen über allergische - 97:

156

Carcinoid (v. Hydroxytryptamin) 92 :

62(A), 203; 95: 209

-, metastatising tumours 85: 168

-, vom pathologisch-anatomischen Standpunkt aus 95: 169

-, Serotonin und das - Syndrom 95: 189

-, Serotonin Wirkung auf Azidität des Magens und auf Motilität des Ver-dauungstrakts 91: 254

-, Darminhalt: Über die Art in der

schwach sauren Zone wirksamen

Protease des Magens und über ihr

Vorkommen im - 81: 44

-, ententes 76: $369(\mathrm{~A})$; 79: $163(\mathrm{~A})$;

80: 303 (A); 82: 186 (A)

-, acute gastro -, as problem of circulation and permeability 65: 149

-, -, Röntgenbefunde - bei verschie-denen Formen 68: 43

-, chronic, clinical observations on patients with $-70: 65 ; 88: 73$

-, - without colitis 82: 129, 210

- $\quad$ colitis Breslau (Salmonella typhi

murium) mit tödlichem Ausgang

87: 158

Index rerum ad vol. 64-100

295

Intestinum tenue

-, nec Гoticans 83: 248 (A)

,-- , post-operative necrosis of intestinal mucous membrane 81: 74

Gefäße, intramurale Verlauf der -

66: 249

Veränderungen bei Hepatitis epide-

mica 70: 24

Ileus 75: 49 (A); 76: 377 (A); 80: 309

(A); 98: 244 (A) biliaire 76: 37 (A) Darmverschluß und sonstige Weg-störungen des Darmes 84: 379 (L) -, akute Erkennung und Behand-

lung 88: $131(\mathrm{~L})$ - Frage des arterio-mesenterialen

- 79: 101 dietetically induced, experimental- 99: 22

après gastrectomie 66: 174

spasmodique à forme «d'intestin de poulet» 69: 302

-, spastischer infolge Harnverhaltung

77: 348 -, occlusion aiguë 75: 390 (L) -, -, équilibre humoral des - avant

Tintervention 80: 151 -, -, radio-diagnostic 83: 258 (L) invagination intestinale 64: 299; 76: 253 (L)

Motilität Untersuchung mít Intesti-nalsender 93: 145

des Darmtraktes, Untersuchungen

über die - 86: 80

-, gastrointestinaler Durchgangzeit

und Auerbacher Plexus 100: 137 -, smooth muscle properties of intestinal - 85: 130 -, -, stimulating substances in intestinal wall 87: 10 -, effect of analgesics and of electrolyte changes on - 85: 142 Neoplasmata 76: 375 (A); 80: 382 (A) -, Hämangiomatosis 93: 357; $98: 321$-, small - $79: 343$ (C); 345 (C) -, sarcomes primitifs du jéjuno-iléon 96: 69 (L) -, Physiopathologie 98: 191 (L)

-, Radiologia 70: 113; 76: 343; 77: 328 (L); 82: 37 (E), 250 (L) -, Bariumsulfat-Emulsion Guerbet in

der Röntgendiagnostik des -

94: 251 -, Radiokinema des - 92: 283 -, Sorbitol, action dans Гexploration

radiologique clinique du - 94: 269

(L) -, Spezialtische, röntgenologische Untersuchung der untersten Darmab-schnitte mittels eines - 86: 1 traitement des maladies chroniques 98: 127 (L) tuberculous disease of megaloblastic anaemia and subacute combined degeneration from - 79: 342 Volvulus 75: 172 (A) Verdauung, histophysiologische Untersuchungen am Darmpithel wäh-rend der - 88: 133 
Iron: v. Ferrum

Iproniazid associated with hepatic necrosis 98: 273 (C)

Isoniazide, traitement des rectocôlites hémorragiques par-94: $258(\mathrm{~T})$

Jaundice: v. Icterus

Jejunum 84: 139 (A); 98: 187 (A)

-, Diverticulosis, schwere - jejuni als Teilursache des Spruesyndroms 75: 353

,-- of $-80: 377$ (C)

-, - an Flexura duodeno-jejunalis 66: 90

-, Geschwiirsbildung im Magen, Duodenum und - 74: 186 (L)

-, Fistel, Hypoproteinämie infolge ga-stro-jejuno-kolischer - 72: 289

,-- jéjuno-colique secondaire à tu-meur maligne du colon descendant 67: 113

-, function, changes after partial gas-trectomy 95: 1

- , jejunitis, dumping syndrome, hypo-glycemia and - 79: 16

296

Index rerum ad vol. 64-100

Jejunum

,-- and mesenteric lymphadenitis, symptomatic sprue due to nonspecific chronic - 80: 283 -, ulcères, perforations en péritoíne libre

87: 1 -, Varicosis - mit schwerer sekundärer Anämie 81: 228 (C)

Journées: v. Societates

Kaffee-Bohnen und - Ersatzmittel, Wir-

kung auf Magenfunktion 68: 182 Kalium und Kohlenhydratstofiwechsel

bei Diabetes mellitus 76: 276 Kaliémie, hypo - par abus de laxatifs

96: 260 (E) Kalzium: v. Calcium Kathepsin, Biologie des Magen - 73:

320 (L) Keimdrüsen: v. Ovar Kinder: v. Pediatria Kohlensäuregehalt der wandständigen

Zellen menschlicher Hohlorgane 89:

101 Kolloquium: v. Societates Konjonktivitis, chronischen Blepharo -,

intestinale Autointoxikation als Ur-

sache schweren - 67: 147 Krebs: v. Carcinoma Krieg: v. Bellum

Laboratorium praxis 94: $132(\mathrm{~L})$

Lactum

-, Milch und -produkte in der Ernäh-

rung und Diätetik 89: 150 (L) -, in diverticula of colon 100: 61 (T) -, Milk-Allkali-syndrom 84: 259 (E)

Lambliasis: v. Parasiti

Laparoskopie, Leitfaden und Atlas der -

99: 275 (L) - und Gastroskopie 78: 68 (L) -, Lehrbuch und Atlas der - und Leber-

punktion 98: 125 (L) -, Komplikationen bei - 91: 375

- cholangiographie - 79: $62(\mathrm{~L})$

-, photographie $-82: 88$

Largactil: v. Chlorpromazine

Laxatif qui obstrue le tube digestif 78: 372 (E)

-, cellulose, significance of intestinal decomposition products of cellulose on the effect of - 82: 10

-, hypokaliémie par abus de - 96: 260 (E)

Leber: v. Hepar

Leucine-amino-peptidase

in serum 95: 57

-, in internal diseases 99: 237

Leukoplakia 85: 175

Leukotaxine in peritoneal free fluid of

experimental pancreatitis 78: 353 Lipasespiegel im Blut 96: 343

Lipid, acides gras, teneur des - alimen-

taires en 87: $114(\mathrm{E})$-, résorption et transport des acides gras

74: 189 (L) -, fat mobilization and its significance

for treatment of obese patients 89:

109 -, fetthaltige Ergüsse der Körperhöhlen,

Diagnose und Pathogenese 74: 42 -, Fettsäuren Stoffwechseluntersuchun-

gen mit einem Mischglyzerid der -.

I. Ausnützung im Darm. II. Verände- 
rungen des Ketonkörpergehaltes 91:

89, 199 -, foie et transport des graisses 80: 1 -, émulsionnants, influence sur résorption des graisses dans Гinsuffísance pancréatique 78: 241 -, contribution à $\Gamma$ étude du transit à

l'aide de la tripalmitine-14C 98:393 (L)

Lipoide, angeborene Störungen im Stoff-wechsel der - 97: 247

Lipotropes, substances - dans prevention de lipophanérose du foie 90: 329

Loop-syndrom, postgastrectomy mega-loblastic anaemia and - 89: 326

Index rerum ad vol. 64-100

297

Lupus erythematodes visceralis gastro-

intestinalen, Symptome beim - 95:

155 Lymphogranulomatose solitaire de 1'es-

tomac 86: 531 Lymphogranuloma venereum, Ano-rec-

to-colonic - 97: 89

Magen: v. Stomachus Magen-Darmkrankheiten: v. Gastro-

enterologia Maigreurs 71: 192 (L) Mastdarm: v. Rectum Melanose der Lippen- und Mundschleim-

haut mit gastro-intestinaler Polypose

84: 341

Medizio, innere, Differentialdiagnose

100: 272 (L) -, -, Therapie Fibel der 98: 391 (L)

Mercure: v. Hydrargyrum

Metabolism

-, Stoffwechselkrankheiten, cerebrale

Manifestationen hereditärer 97: 293 -, -, Störungen Genetik der - 97: 226

- mineral et fonction parathyroïdienne

64: $172(\mathrm{~L})$

-, Stoffwechsel - und Verdauungs-Krankheiten 77: 191 (L)

Météorisme abdominal 66: 130, 141

Méthionine et 35S-Méthionine, absorption intestinale au cours des stéatoses et des cirrhoses du foie 98: 393 (L)

Milch: v. Lactum

Milz: v. Splen

Morphine, effect of - sulphate, on stomach after gastroenterostomy and partial gastrectomy 81: 110 test cholérétique dans les affections

des voies biliaires et migraines 97: 137

Mouth: v. Os

Mucinases in ulcerative colitis 76: 346

Mucoprotéines, intérêt des - en patholo-

gíe digestive 98: 307 (E) Mund: v. Os

Nebenniere: v. Surrenal

Neoplasmata

-, tumeurs digestives, dépistage systé-

matique 80: $181(\mathrm{E})$-, gutartige Tumoren 100: 42

Nephrotischen Symptomenkomplex, Ei-weiß-Stoffwechsel beim - 69: 121

Neurologia

-, cerebrale Manifestationen hereditärer

Stoffwechselkrankheiten 97: 293 -, Gehirn, Auswirkung chronischer Le-

berkrankheiten auf das - 90: 259 -, infiltration novocaïnique préfrontale

en pathologie digestive 83: $187(\mathrm{~T})$-, - du cerveau préfrontal. Guérison immediate d’un ulcère gastrique géant 82: 181 (T) -, role du système neuro-végétatif en

pathologie gastro-intestinale 72: 420

(L) -, Neurinome des Magendarmkanals 91:

266 -, Neurodigestive asthenia 75: 228 -, neurohormonale, syndromes digestifs

et pathologie - 70: 223 (L) -, neurologische Aspekte der akuten Por-

phyrie 97: 384 -, Neurome und Neurofibromatose nach

Untersuchungen am menschlichen

Magendarmschlauch 74: 188 (L)

Niere, Einfluß von Pankreastumoren auf 
die - 64: 324 Noble, operation de -, dans le traitement

des adhérences abdominales 77 :

303 (E)

Nutritio (v. Alimentatio, Carences)

-, maladies de la - 64: $160(\mathrm{R}) ; 65: 379$

(R); 68: 103 (A); 69: 332 (A); 71:

376 (A) -, dénutrition 75: 59 (L)

chez malade chirurgical 76: 29 (T)

and haematological effects of operation on the stomach 79: 337

-, - effects of oral antibiotics 79: 327

298

Index re $\pi$ im ad vol. 64-100

Obesitas 64: 145 (R); 68: 333 (A); 70: 347 (A); 82: 367 (A); 88: 68 (L)

-, Abmagerungskur nach Antoine 72: 311

-, obese patients, fat mobilization treatment 89: 109

Obstipation, v. Constipation Oddi, sphincter: v. Choledocus

CEdèmes 95: $137(\mathrm{~L})$ - de famine et carence alimentaire 68: 169

(Esophagus

64: 263 (C), 381 (L); 65: 284 (A); 66: 43 (A), 94 (A), 234 (A); 67: 78 (A), 249 (A); $68: 158$ (A); 69: 90 (A); 70: 49 (A); $71: 125$ (A); $72: 74$ (A); $73:$ 108(A);74:112(A);76:58(A);78:259 (A); 79: 58 (A), 380 (L); 80: 63 (A); 81: 58 (A); 82: 53 (A); 83: 137 (A); 84:77 (A); 85:60 (A); 86:57 (A); 87:54 (A); 88: 264 (A); 89: 58 (A); 90: 56 (A); 91: 131 (A); 93: 60 (A); 94: 123 (A); 95: 64 (A); 97: 208 (A); 99: 67(A) -, Achalasia (v. Therapia) 100: 87,157 brachyæsophage 76: 48 (A); 86: 206 Cardiospasm 75: 259 (E); 76: 49 (A); 78: 342 -, achalasia, nature 86: 187

observations of the cardia $86: 178$

physiology and treatment 92: $114-$, motility and pharmacology of the

esophagus in - 86: 174 -, psychopathology and psychotherapy 86: 211

65: 341 und Ulcus pepticum oesophagi

-, méga-cesophage 76: 46 (A) -, étiologie et traitement 86: 200

- $\quad$ et hernie hiatale, péristaltisme æso-

phagien 86: 151

-, Therapia

-, -, dilatation in treatment of achalasia - 86: 210 -, -, -, Heller's operation for achalasia of the cardia 85: 59 (T); 89: 323

,,,-- cardiomyotomy, competence of

cardia after - 92: 123 , -, -, traitement du méga-cesophage par cardiospasme 68: 110 (L); 86: 179, 208 Carcinoma 76: 53 (A); $83: 135$ (A); $99: 401$ (L) , -, eine mögliche Hilfsmethode zur

Frühdiagnose 96: 371 l -, Röntgentherapie des - 65: 214 , -, traitement chirurgical du - et du

cardia 79: $117,-$, traitement chirurgical par exérèse,

résultats éloignés 84: 292 , columnar epithelium 86: 183 , Diverticules 76: 48 (A), -, Chirurgie des Speiseröhren -

88: 72 (L), Dysphagia and short - 73: 186 (E) , - in Felty's syndrome 75: 129 l-, dyskinésie of the - 86: 173 , -, syndrome de Kelly-Paterson (formes frustes) 86: $167,-$, etude radiologique des - (espagnol)

86: 212 , endoscopie, histologie et radiologie de $\Gamma$-dans affections hépatiques 86: 266 , Fremdkörper in der Speiseröhre und im Magen 65: 172 (C) ,

Neoplasmata , -, léiomyome 92: 257 (C), -, Lymphogranulom, malignes mit

primärem Befall des - 98: 281 ,-, tumours of the - 97: 59 (L) CEsophagitis peptica 86: 304 -, marginal ulceration and peptic

ulcer of the - 86: 268 -, Ulcus pepticum oesophagi 76: 52

(A); 83: 131 (A) - und Kardiospasmus 65: 341 deformity of lower end - in pernicious anaemia 79: 376; 85: 223 -, gastro-æsophageal junction, non malignant disorders 78: 333 -, lower oesophageal ring 99: 217 -, Pathologie der Cardia - 89: 38 Physiology 89: 1

Index $\Gamma$ rum ad vol. 64-100

299

(Esophagus

-, -, esophageal motility in patients with scleroderma and related diseases 86: 763 -, -, swallowing mechanism 78: 347 -, -, pression intra-oesophagique et intragastrique 93: 46 -, Radiologie des affections non-cancé-

reuses de - 86: 257 -, -, study of movements of the tongue, pharynx and - in acrosclerosis 79: 361 -, -, cineradiography of the symptomatic - 86: 162 -, -, study of some neuromuscular abnormities of the - 86: 164 -, Rupturen und Perforationen der

Speiseröhren 76: 55 (A); 80: 364 - Stenosis (v. Cardiospasm, Neoplas-mata) -, Atresien beim Neugeborenen, Be-

handlung 79: 146 -, caustic strictures, treatment 87:

405 (T) -, obstruction - in childhood 86: 301 
cardia, par cicatrice d'ulcus 65: 277 (C)

cesophagienne bénigne (espagnol) 86: 317

-, non malignant surgery 86: 283 Therapia, chirurgie de $\Gamma$ - et du cardia 79: 138, 254 (L) -, esophagoplasty, modern concept

86: 311 -, Operationen am - 82: 249 (L) -, Plastik, Erfahrungen mit der an-

tethorakalen $-84: 301-$, resection, contrôle radiologique 85 :

329 Tuberculosis 83: 136 (A) Ulcus pepticum oesophagi: v. Oeso-phagitis peptica $\Lambda^{1 / 8}$ rices 76: 51 (A); $83: 135$ (A)

æsogastriques, diagnostic; valeurs

respectives du transit baryté et de

la splénoportographie - 89: 83

-, secondary to portal cirrhosis 80: 257

,-- , rupture, anastomoses portosysté-

miques pour hémorragies 98: 87 -, -, traitements nouveaux 72: 62 (E)

Ornithose mit hepatitischem Bild 98: 15

Os (v. Dentes, Lingua, Saliva)

65: $102(\mathrm{~A}) ; 67: 78(\mathrm{~A}) ; 68: 72(\mathrm{~A})$;

70: 49 (A); 71: 125 (A); 72: 73 (A);

74: 110 (A); $78: 67$ (A); 79: 57 (A) -, spez. Pathologie und Verletzungen

71: 256 (L) -, Mundschleimhaut, Krankheiten der

Lippen und - 94: 133 (L) -, stomatites 75: 60 (L)

Osier, Morbus -, und Cirrhosis hepatis

teleangiectatica 83: 157 Osteitis deformans Paget, position of

hepatopathy within the etiology of -

64: 84 Osteoporosis and arthrosis, relationship

with vitamin A metabolism 65: 51

Ovarial-Funktion in Geschwürkrankheit

93: 294 -, Keimdrüsenhormonen in Behandlung

des Magen- und Zwölffingerdarmge-

schwürs 66: 343 (R)

Pancreas

65: 124 (A); 66: 110 (A), 246 (A), 357 (A); 67: 101 (A); 68: 99 (A); 70: 109 (A), 213 (A); 73: 304 (A); 78: 73; $80: 392$ (A); $82: 62$ (L), 379 (A); $84: 264$ (A);

86: 64 (L), 812 (A); 87: 122 (A); 89: 384 (A); 93: 259 (A), 325 (A); 98: 127 (L); 99: 201 (A); 100: 207 (L)

-, pancréato-biliaires, correlations hé-pato - 83: 243 (E)

-, Chirurgia 77: 326 (L); 78: 127; 88: 268 (L)

,-- , anastomoses bilio-digestives et pan-créato-digestives 99: 78 (L)

-.Diagnosis 80: 392 (A); 82: 308 (A); 83: 376 (A); 86: 811 (A)

-, -, pancreatic function 74: 321, $360(\mathrm{E}) ; 99: 279$

300

Index rerum ad vol. 64-100

Pancreas

-, -, funktionelle - mit besonderer Be-rücksichtigung chronischer Pan-kreasafFektionen 69: 233 -, -, äußere Störungen, Sekretion des -beim frischen

Zwölffingerdarmge-schwür 88: 83 -, -, secretion pancréatique au cours certaines affections hépato-biliaires 72: 51 -, -, Sekretionsstudien am - 89: 387 (L) -, -

, Pruning der äußeren -funktion mit geeigneter doppellumiger Sonde 81: 225 -, -, Pruning Pankreasfunktion mit ver-einfachter Fermentbestimmung 81: 206 -, -, Funktionsprüfung, verbesserte Me-thode zur Bestimmung der proteo-lytischen Fermente im Duodenal-inhalt 82: 1 -, Diasteswerte im Urin und Blutse-rum unter bestimmten Ernäh-rungsbedingungen 66: 12 -, Funktionsprüfung zur Bestimmung der stärkeverzuckernden Fermente im Duodenalinhalt 80: 211 -, pancreozymin, use of - in man 86:

463 -, - and uropancreozymin, comparative electrophoretic study 94: 290 -, Prostigmin test, diagnostic value in chronic pancreatopathie 97: 21 -, Trypsin, fluorimetrische Dosierung

100: 209 -, secretin and pancreozymin in man

86: 463 -, secretin test of - function and its

use in steatorrhea 74: 257 -, Sekretin und Äther, Reizeffekt auf exkretorische Pankreasfunktion 96: 362 -, épreuve à la Sécrétine, insuffisance pancréatique externe étudiée par -78: 169 et duodenum 89: 389 (L) Enzymologie, Leber und - 98: 125 (L)

Erkrankungen, pathologisch-anato-mische Grundlagen der klinischen -78: 123

exocrine 100: 136 (L), 207 (L) heterotopic pancreatic tissue, chronic peptic ulcer in - 92: 73 Inselsystem des - 79: 254 (L) insuffisance pancréatique, influence des émulsionnants des lipides sur la résorption des graisses 78: 241 internal secretions of the - 88: 69 (L) Pathologie des kindlichen - 86: 128 (L)

Langerhans Islands influence of cor-ticosterone and the liver upon the stimulation of cells in - 94: 295 Neoblastoma 82: 313 (A) -, carcinoma 83: 378 (A) -, -, Chirurgie des - 78: 152 -, - mit Hyperkalzämie 96: 197 -, islet tumors silvercells in -

71: 193 -, Insulome 82: 314 (A) -, - with peptic ulcer 93: 157 
-, Tumoren, Einfluß auf die Leistungs-fähigkeit der Niere 64: 324

Pancreatitis 80: 395 (A); 82: 311 (A);

83: 377 (A); 86: 811 (A); 94: 65; 99:

$16(\mathrm{~h})$

-, experimental leukotaxine in the peritoneal free fluid 78: 353

-, role of disease of liver and biliary tracts in - 80: 263

-, traitements des - 84: $72(\mathrm{~T})$

380; 93: 129

acuta, ACTH in treatment of -87 :

, corticothérapie 84: $73(\mathrm{~T}) ; 94: 52$

(T); 97: $51(\mathrm{~T})$

-, Entstehung durch viscero-vis-

cerale Reflexreaktion 81: 36

- $\quad$-, Enzymintoxikation bei - 95:

60(E)

-, Entstehungsmechanismus des

Kreislauf kollapses bei -, 84: 225

- als Folge generalisierter Arteri-

tis $72: 81$

Index rerum ad vol. 64-100

301

Pancreas

,,--- nouveaux aspects cliniques 71: 257

-, enzymes sériques 95: 73

- mortelle après drainage trans-papillaire du canal cholédoque 88: 311 (C)

64: 73 chronica, Diagnose der latenten -

- $\quad$ - mit Hyperfunktion der Langer-

hansschen Inseln 66: 72

Diagnose 78: 205

-, duodenal diverticula as the cause of - 80: 203

-, pancreatopathy, trophopatic 99: 145

-, relapsing - diabetes mellitus and gallbladder disease 79: 282

- gauches 70: $55(\mathrm{~L})$

- syndromes - 71: 127 (L)

74: $187(\mathrm{~L})$

,-- etude clinique et radiologique

Physiologia 82: 375 (A) pseudocyst, cystography in pancreatic - 99: 164 (C)

Radiologie, nouvelle manière 700: 199 (E)

Röntgenbefunde bei Pankreaserkran-kungen 78: 93

Stein, solitär des - 81: 336 (C) therapy, substitution dosage schedule in pancreatic insufficiency 93: 324 (T)

Paraffine, huile, nouvel inconvenient de

$$
\text { 74: } 296(\mathrm{E})
$$

Paramyloidoses, Corino de Andrade -

99: 118

Parasiti

68: 337; 69: 215; 71: 249; 75: 125, 169; SO: 250; 82:181; S3: 313; 85: 351 -, Anguillula intestinalis 74: 150 (C) -, Amibiasis 75: 42 (A); $76: 252$ (L); 80:

312 (L) -, - autochtone 79: 319 (L); 100:

125 (R) -, - cæcale pseudo-tumorale 74: 319 (L) -, - expérimentale 100: 125 (R) -, -, amibophobie 89: 263 (E)

-, amæbicide synthétique susceptible de remplacer Гémétine, la 2-dé-hydro-émétine 97: 136 (T)

-, dysenterie amibienne,constatations au cours d'une épidémie 70: 82

-, intestinal und duodenal Syndrom 91: 42

Ascaridiase et radiographie 67: 64 (C)

Balantidienkolitis 64: 12

Bilharziasis, hepatic - und Spleno- 
portographie $93: 86,167$

Chilomastiase 71: 410 (E); 91: 410

et éosinophilie 79: 44 (E)

filagellés intestinaux, etudes morpho-

logique et biologique 64: $382(\mathrm{~L})$

Lambliasis 75: 42 (A); 96: 115

observations cliniques 66: 323

-, Therapie 65: 24; 71: 254 (T)

parasitären Erkrankungen, Klinik

der - 94: 131 (L)

Tabulae parasitorum intestinorum 85: 126 (L)

treatment of parasitic infections 86: $804(\mathrm{~T})$

des parasites intestinaux par Гоху-

gène, le melange éther-oxygène et

le protoxyde d'azote 94: $53(\mathrm{~T})$

Parathyreoidea 64: 152 (A); 65: 128 (A) -, fonctionnement dans Гulcère gastro-duodénal 98: 193 et métabolisme mineral 64: $172(\mathrm{~L})$

Peau: v. Cutis

Pediatria, Kindesalter, gastroenterolo-gische Probleme im - 90: 312; 100: 133 (L)

muqueuse digestive en - (espagnol)

99: 143 (L)

Pentolyse, application au diagnostic du

cancer 78: 192 Pepsin inactivation in ulcer therapy 70:

53 (T) Pepsinogen in blood and urine 98: 319

(L)

Peritoneum

65: $118(\mathrm{~A}) ; 78: 267$ (A); 83: $256(\mathrm{~L}) ; 88: 264$ (A)

302

Index rerum ad vol. 64-100

\section{Peritoneum}

-, Resorption von Enzymen durch -97: 8

-, peritonitis, bile following needle biopsy of liver, recovery from - 80: 357

-, -, eitrige - bei Diverticulitis perfora-tiva 87: 85

-, pneumo -, diagnostic 85: 213

-, -, eine Nadel für gefahrlose - 96: 150

,-- retro - 79: $319(\mathrm{~L})$

-, Talkgranulomatose des - 73: 129

Peutz's syndrome 86: 456; 97: 119 (C) Pharmakologie des Verdauungsappara-

tes 66: 213 (R) Phosphatase alcaline, experimental investigation in serum 92: 78 Physiologie, klinische 96: 67 (L) Pituitary gland: v. Hypophyse Placebo, effect in peptic ulcer and other

gastroduodenal disorders 94: 11 Plasmocytom, ist das maligne - eine pri-märe Eiweißstoffwechselstörung ? 75: 238

Podagra 65: 304 (A) -, hémorragies sine materia dans -100: 53

Polysaccharid: v. Glucids

Porphyrien, Klinik 97: 370

akuten, neurologische Aspekte 97: 384

problematik aus dermatologischer Sicht 97: 404

Porta, Vena 85: 383 (L); 86: 64 (L)

porto-cavaler Beurteilung des Pfort-aderhochdrucks vor und nach - 87: 209

Anastomose - systémique pour hé-

morragies par rupture de varices æsophagiennes 98: 87 -, hypertension portale 81: 364 (L); 96: 66 (L)

81: 293 and hypotension in the portal system

-, -, exploration radiologique et mano-

métríque de $\Gamma$ - 82: $65-,-$, costal intra-osseous Venography in

the diagnosis of $-88: 21 ; 98: 146$

-, thromboses portales 75: 65; 98 :

394 (L) -, Zirkulation, tierexperimentelle Un- 
tersuchungen 94: 102

Potassium: v. Kalium

Prednisone: v. Cortisone

Proctitis: v. Rectum

Professions traumatisantes, notes sur -

dans genèse ulcère gastroduodenal

67: 30 Prognose chronischer Erkrankungen 97:

55 (L) Prostigmin test in chronic pancreato-

pathies 97: 21

Protids

des Menschen 82: $62(\mathrm{~L})$

-, Dysproteinämieentstehung bei Leber-

erkrankungen 95: 88 -, Rhodan-Eiweiß-Relation im Speichel

und Magensaft für Nachweis «patho-

logischer»-85: 313 -, Kost beim Sport 65: 244 -, Stoffwechsels, angeborene Störungen

97: 275 -, Untersuchungen am Blutserum

72: 4 -, Hypoproteinämie in gastro-jejuno-

kolische Fistel 72: 289 -, Nahrungseiweißes, Beziehung des -

zum Ablauf des BetriebsstofFwechsels

65: 308 (L) -, Plasma - and gastro-intestinal tract

100: 135 (L) -, saving effect of androgenic hormones

in treatment following total gastrecto-

my 93: $324(\mathrm{~T})$

Psammo-carcinome des ovaires qui évo-lue depuis 18 ans 71: 315 (C)

Psychical

-, influences in gastro-enteric phenomena 89: 125 (E)

- psychosomatic aspects of ulcerative

colitis 86: 591

- et gastro-entérologie 99: 277 (L)

Punktion, Organ- in der klinischen Dia-

gnostik 84: 379 (L)

Index rerum ad vol. 64-100

303

Purgatio partialis et generalis durch sub-aquale Darmbäder und kombinierte Diät 65: 258

Quincke's disease, gastroscopically verified oedema of stomach of familiar -65: 129

Radiotherapy: v. Röntgen

Rate: v. Splen

Recto-côlite hémorragique et purulente:

v. Colitis ulcerosa Rectoscopie: v. Rectum

Rectum

67: 110 (L); 71: 247 (A); 75: 175 (A);

79: $180(\mathrm{~A}) ; 82: 378(\mathrm{~L}) ; 85: 237$ (A);

87: 117 (A); 94: 133 (L), 326 (A) -, Atresia als Grand eines unvollstän-

digen Megakolon bei 70Jährigen

66: 28 (C) -, carcinome, chirurgie 92: 297 -, -, heterotopic ossification in - 98: 48 -, -, electro-coagulation - 85: 117 (T) -, -, radiothérapie de contact 92: 287 ,

302 -, - traitement par radiothérapie de contact 71: 378 (T); 91: 412 (T); 98: 319 (L) -, invagination sigmoïdo-rectale 79:

306 (E) -, Lymphogranuloma venereum 97: 89 -, polyps of colon and adenomatous as

precancerous lesions 86: 370 -, melanosis proctocoli 64: 68 -, Procto-colitis idiopathic 93: 107 -, Rectite hémorragique 76: 361 (E); 87 :

225; 93: $107-,-$, granulomateuse sténosante non

spécifique 81: 318 -, -, traitement des - 64: 382 (L) -, Rectoscopies, principes 84: 381 (L) -, -, Okklusionsblatt für - 64: 91 -, -, routine for early detection of rectal carcinoma 79: 347

-, Selbstverschädigungen seltener Lo-kalisation 99: 247 (C)

Regimes: v. Diaeta

Reflexuntersuchungen am Verdauungs-kanal mit Pantocain 77: 354

Réserpine et poussée evolutive d'ulcère duodenal 92: $181(\mathrm{~T})$

-, effect upon basal gastric secretion 90: 401 (T)

-, antagonisme entre - et inhibiteurs de la mono-amino-oxydase 98: 217 
-, induced gastro-intestinal hemorrhage 96: 395 (T)

-, lesions gastriques post-, role du cortex surrénalien 98: 225

Résines, emploi des - va-t-il remplacer le regime déchloruré ? 78: 257 (E)

- $\quad$ synthétiques dans traitement ulcère

gastro-duodénal 75: $112(\mathrm{~T})$

-, Untersuchungen mit «Cellumikro-moor» 83: 343

Rheumatismus, Herdinfekt und -

66: 55 (L) -, Arthritis bei chronischer ulzeröser Ko-

litis 99: 374 -, sind anti - Präparate für den Magen

gefährlich? 85: 50 (E)

Röntgen (v. Barium)

-, gastric irradiation in the treatment of

duodenal ulcer 87: $345(\mathrm{~T})$-, Radiothérapie abdominale et ulcère

face antérieure estomac 91: 35 (C) -, - contact dans cancers ano-rectaux 71: 378 (T); $91: 412$ (T); $98: 319$ (L) -, Diagnostik der Leber $94: 129$ (L) examinations in acute abdominal dis

eases 95: $399(\mathrm{~L})$

-, Differentialdiagnostik 77: 326 (L) -, digestive tract in - 81: 363 (L) -, Probleme, gastroenterologische - 95: 398 (L)

Salazopyrin, Behandlung der ulzerösen Kolitis mit - 66: 312

304

Index rerum ad vol. 64-100

Saliva, parotid gland and diabetes mel-

litus, swelling of- 68: 139 -, parotidite asymptomatique de carence

83: 366 (E) -, Speicheldrüsen des Menschen 94:

131 (L)

Sanguis (v. Anaemia, Haemorrhagia) -, Bildung und Verdauungssäfte 66:

116 (L) -, Plasma-Zellen bei Hepatitis epide-

mica 71: 97 -, Zusammensetzung, Beziehungen zwi-

schen verschiedenen Magenaffektio-

nen und Veränderungen 64: 348 (R) -, group ABO secretion and duodenal

ulcer families 92: $99-,-$ in non gastroenterologic diseases

92: 92 -, -, in gastro-intestinal diseases 92: 95 -, - antigen in gastro-intestinal disease 92: 103

and anthropology 92: 58 (E)

-, groupes sanguins et maladies de $\Gamma$ es-

tomac 82: 238 (E) -, haemopoiesis, relation to disorders of

alimentary tract 79: 322 -, appendicite et diarrhée sanglante 95:

121

Scorbutic vertigo 71: 129

Sex

-, Hormone als Heilmittel innerer

Krankheiten 67: 331 (L) -, testosterone à haute dose dans le trai-

tement de la cirrhose atrophique et

ascitique (ital.) 86: 803 (T)

Sigmoïdoscopie: v. Rectum

Skin: v. Cutis

Skoliose, Kypho- bei Anämie 66: 197

Societates

American Gastroenterological Association 1950 (Atlantic City) 75: 358

Association européenne de Gastro-enté-rologie 1948 (Lausanne) 73: 321

1950 (Madrid) 76: 221952 (Bologne) 79: 45

1954 (Paris) 82: 39

1956 (Londres) 86: 133, 379, 542

Association d'Etudes physio-pathologi-

ques du Foie

1955 (Geneve) 83: 353, et (Vichy)

84: 195

British Society of Gastroenterologists

72: 241 
(London) 73: 273

(Birmingham) 75: 17

(London) 76: 94

76: 357

(Leeds) 78: 333

(London) 79: 321

(Edinburgh) 81: 65

(London) 83: 1

(Oxford) 85: 129

(Glasgow) 89: 281

(London) 92: 91 Conference internationale d'hépatologie

1957 (Perugia) 88: 127

VIe Conference de pathologie géogra-phique

1956 (Paris) 88: 62

Congrès espagnol de pathologie digestive

1951 (Santander) 78: 255 Congrès international de la fonction bi-

liaire

1958 (Vittel) 90: 393

Deutsche Gesellschaft für Verdauungs-und Stoffwechselkrankheiten

1950 (Bad Kissingen) 76: 224

1957 (Bad Kissingen) 90: 129

(Kassel) 95: 141

(Zurich) 96: 73

(Hamburg) 97: 217 Journées des gastro-entérologues fran-

çais

1951 78: 56

1953 80: 240

1955 85: 52

1957 89: 118

1961 98: 118

Journées méridionales de gastro-entéro-logie 1955 (Marseille) 85: 228

Index rerum ad vol. 64-100

305

Societates

Kolloquium über Leberkrankheiten

1955 (Bad Bertrich) 86: 795 Schweizerische Gesellschaft für Gastroenterologie; Société suilsse de Gastro-

entérologie

1940 (Biel) 66: 38

(Zurich) 68: 69

(Basel) 69: 83, et (Lausanne) 208

(Aarau) 70: 43

(Chur) 71: 104

(Geneve) $71: 338$

(Bern) 72: 341

(Lugano) 75: 109

(Berne) 77: 105

(Fríbourg) 78: 197

(Zurich) 79: 151

(Geneve) 80: 167

(Brunnen) 82: 125

(Basel) 84: 372

(Lausanne) 87: 278, et (London) 88: 269

(St. Gallen) 89: 261

(Bern) 92: 201, 3821960 (Zurich) 96: 73 
Schweizerische Gesellschaft für innere

\section{Medizin}

1950 (Neuchâtel) 76: 24 Société beige de Gastro-entérologie

(Bruxelles) 76: 100

(Anvers) 77: 171

79: 49

(Bruxelles) 80: 175

(Gand) 82: 46

(Bruxelles) 83: 362

(Bruxelles) 85: 342

(Liège) $87: 397$

(Bruxelles) 91: 571960 (Bruxelles) 95: 392

Société Internationale de Gastro-entérologie 1954 (Paris) 81: 180

Swedish Society of Gastroenterology 1958 (Eskilstuna) 93: 107

Sondenbehandlung chronischer Magen-und Zwölffingerdarmgeschwüre 82: 63 (L)

Sorbitol, polyvalence du - 96: 390 (E)

-dehydrogenase bei Lebererkrankun-gen 96: 26

dans Гexploration radiologique clini-que de Гintestin grêle 94: 269 (L)

Sous-alimentation: v. Alimentatio, Ca-

rences Speiseröhre: v. Oesophagus Spirolactones dans le traitement de la

cirrhose hépatique 97: 205 (E)

Splanchnic circulation after haemorrhage 81: 12 -, - experimental studies 81: 71 -, Chirurgie 68: 111 (L)

stase circulatoire, troubles viscéraux et thromboses 75: 90

-Gebiet, Stauungszustände im -, vis-zerale Störungen und Thrombosen 75: 74

Splen

-, Milzblutungen, zweiseitige 73: 30

-, rate et ses maladies 83: 138 (L)

-, Splenektomie, Indikationen 94: 334

(L) -, splénoportographie et splénomano-

métrie (ital.) 89: 279 (L) -, - valeurs respectives du transit ba-ryté et de la-pour le diagnostic des varices cesogastriques 89: 83

in hepatic Biharziasis 93: 86, 167

Sport, Eiweißkost beim - 65: 244

Steatorrhea (v. Ileum, Feces, Intestinum

tenue) -, über das Auftreten von Herterscher

Krankheit und Allergien in der glei-

chen Familie 96: 111 -, Klinik des Malabsorption-Syndroms

97: 313 -, fat fractions in stool of - 93: 27

- bleu de Nil, reaction, comme moyen

d'appréciation rapide de la teneur en

graisses des matières fécales 78: 249

-, zur Diagnostik der - 83: 224 -, Sprue 76: 372 (A); 79: 163 (A); 80: 306 (A); 82: 186 (A)

25 Gastroenterologia, Vol. 100, No. 5/6 (1963)

306

Index rerum ad vol. 64-100

\section{Steatorrhea}

,,-- symptomatic, due to nonspecific

chronic jejunitis and mesenteric

lymphadenitis 80: 283 -, -, à propos d'un cas de - 64: 177 (C) -, -, studies on the aetiology of the -

syndrome 78: 357 -, -, stéatorrhées gastro-intestinales en

dehors de la-80: 101 -, -, schwere Diverticulosis jejuni als

Teilursache der Spruesyndroms 75 :

353 -, -, pathologisch-anatomische Unter-

suchungen bei 7 Beobachtungen

von einheimischer und symptoma-

tischer Sprue 79: 257 -, einheimischer - 64: 257 (L) -, Vasomotilität bei Sprue und ande-

ren Resorptionsstörungen 85: 1 -, influence of gluten and antibacterial

agents on fat absorption in the 
sprue syndrome 81: 98 -, Enteritis regionalis mit symptoma-

tischer Sprue 81: 1 -, symptomatic - 73: 166 -, Röntgenbild des Dünndarmes bei

einheimischen - 64: 191 -, einheimische - 66: 56 (L) -, röntgenologische Befunde bei Sprue

und enteralen Resorptionsstörungen 97: 357 -, Pathophysiologie der Resorption

bei - 97: 305 secretin test of pancreatic function and its use in - 74: 257 idiopathic - study with 131J 93: 228 Whipple, maladie de 92: 64 (A); $100: 264$ (A)

-, trois cas de - 88: $172(\mathrm{C})$-, Wirkung des Corticosterons bei

idiopathischen $-65: 280(\mathrm{C})$

Stenosen und Atresien, angeborene - des

Magendarmkanals 90: 325 Stoffwechsel: v. Metabolism Stoffwechselkrankheiten: v. Metabolism,

Nutritio

Stomachus (v. Gastrectomie, Gastrosco-

pie, Haemorrhagia, Ulcus pepticum) 65: 112 (A), 372 (A); 66: 44 (A), 117 (L); $67: 78$ (A), 153 (A), 251 (A); 69: 91 (A); $70: 95$ (A); $72: 353$ (A); $73: 127$ (A); 74: 173 (A); 75: 390 (L); 76: 404 (A); 79: 59 (A); 82: 61 (A), 315 (L); 83: 201 (A); 84: 136 (A); 85: 124 (A); 86: 122 (A); 89: 66 (A), 132 (A); $90: 58$ (A); $91:$ 344 (A); 93: 125 (A); 95: 66 (A); 98: 55 (A), 183 (A); 99: 67 (A); 100: 202 (A) Acidité (v. Achlorhydrie, Physiologia)

- $\quad$ test meals, influence of low concen

trations in - 86: 506

-, -, vereinfachte 67: 39; 68: 53

-, indirekte Bestimmung des Magen-

saftes mit Hilfe eines Chininaustau-

schers 85: 20 gastric juice analysis, paper electro-phoretic 86: 384

and saliva, fractionation of human - employing starch electro-phoresis 84: 99

output of $\mathrm{HC1}$ during stimulation of gastric secretion 89: 290

histamin determination in human 79: 185

Magenausheberung, fraktionierte in

der Praxis 89: 165

Magensaft pH-Messungen mit Glas-

elektrode 72: 201

Säureproduktion des gesunden und

kranken Magens, quantitative intra-

gastrale Bestimmung 81: 193

- , secretion gastrique, variations ho-

raires de la - sous fortes doses d'his-

tamine 98: 334 achlorhydrie vraie ou relative 92: 335

- $\quad$ et clinique (ital.) 81: 128 (L)

dyspepsie 84: 133 (E)

Anazidität, Gastroskopische Befunde

bei - 67: 125

Aktionsströme des Magens, Bedeu-tung 79: 227 Anatomie 76: 113 (A) -, arteriography, post-mortem of normal stomach 79: 378

Index rerum ad vol. 64-100

307

Stomachus

-, -, circulation of blood in rat's stomach 79: 356 -, Fundusschleimhaut, histologisches

Bild 84: 103, 273 -, Magendurchblutung 82: 317 -, Magenschleimhaut, Becherzellen in

- 93: 269 -, mucosa, intestinal metaplasia of

the gastric - 86: 353 bezoar, Phyto- 92: 30 (C); 99:123 (C) biopsy, technique 83: 60 -, diagnostischer Wert 84: 1;

100: 19 -, in anaemia with reference to -

79: 329 Dehydrogenase-Enzym-Aktivität-untersuchung am - 84: 164 -, Leitfaden der Gastroskopie und -

99: 77 (L) Magenaffektionen, Beziehungen zwi-schen - und Veränderungen der Blut-zusammensetzung 64: 348 (B) Carcinoma (v. neoplasmata) $71: 237$ (A); 74: 310 (A); 76: 304 (A); 78: 326 (A); 80: 189 (A); 83: 198 (A); 84: 82 (A); 85: 119 (A); 86: 122 (A); 87: 59 (A); 88: 266 (A); $91: 340$ (A); 93: 5; 63: (A); $94:$ 194(A)

aetiology of -, environmental factors 86: 320

pylorique, d'aspect radiologique trompeur: image d'ulcère duodenal 64: 286 (C)

du fornix 75: 1

-, diagnostic précoce 77: 65,103 ;

86: 365 - au debut 64: 382 (L); 77: 73 -, -, cytologic examination of gastric

content 86: 378 -, petit 94: 162; 97: 59 (L)

small, favorable outlook for patient with $-86: 474$ 
stades initiaux du - au bord de $\Gamma$ ulcère 99: 81

-, cyto-diagnostic 85: 190, 257 -, Frühdiagnose zur Hillfsmethode 96: 371

- und Gastritis hypertrophica gi-gantica 93: 133

Entwicklung, Bedeutung der chro-

nischen Gastritis 65: 137

Magenkatarrh, Magengeschwür 67:

$110(\mathrm{~L})$

précancer et tumeur bénigne

86: 508

-, lesions gastriques $86: 350$

-, klinische Bedeutung 86: 359 Therapia 90: 367 -, chirurgische 89: 19

- $\quad$ inoperablen - mit Übertragung

von Magensaft gesunder Menschen

72: 306

-, - mit Autovakzinationen von Magensaft des Kranken 73: 45 Chirurgie 65: 103 (A), 107 (A), 284 (A); $66: 97$ (A), 239 (A), 352 (A), 354 (A); $68: 76$ (A); 69 : 99 (A), 103 (A), 105 (A); 71: 177 (A), 239 (A); 73: 115 (A), 125 (A); 74: 180 (A), 320 (A); 78: 322 (A); $80: 187$ (A); $81: 179$ (A); $82: 190$ (A); $87: 55$ (A) syndromes douloureux de la region épigastrique 66: 117 (L); 74: 120 (L); 79: 62 (L) cytology 96: 65 (L) dilatation, acute 100: 7 diverticula 76: 313 (A); $84: 86$ (A) Druckmessung im Magen-Darmlu-men (v. Physiologia) 84: 252 dyspepsia, studies on functional disorders of the biliary tract 85: 290 Gastritis 74: 190 (L); 76: 116 (A); 78324 (A); 80: 246 (A); 82: 54 (A); 83192 (A); 84: 84 (A); 85: 118 (A); 8868 (L); 91: 277 (A) -, diagnostic biologique 92: 335 et antihistaminiques de synthèse 78: 406 (L)

allergische, akute pseudotumoröse 97: 65

atrophische -, bioptischer Unter-suchungen 83: 203

Cytodiagnostik 65: 229; 82: 117

erosive - 84: 87

Histopathologie 92: 367

308

Index rerum ad vol. 64-100

\section{Stomachus}

-, - Oberflächen 83: 259

cedémateuses et hypertrophiques. Traitement par un gel à base de cortisone 88: $262(\mathrm{~T})$

phlegmonosa, antibiotisch behan-delt 79: 94 (C)

Ulkus und Karzinom 77: 328 (L)

chronica 68: 118 (L); 77: 63 (L); 92: 307; 96: 211,301

-, - und Entwicklung des Magen-

krebses 65: 137 -, -, radiologie 88: 69 (L); 92: 352 -, -, histologie 92: 332 -, hypertrophica gigantea 78: 1; 82:

348; 96: 75; 99: 13(E) -, - und Magenkarzinom 93: 133 -, gastroduodenitis, liver changes in

chronic - 93: 385 -, Gastropathien, diffusen. Beziehun-

gen zwischen Magensekretion und

Schleimhauthistologie 96: 133 gastroskopisches Symptom beim Zwölffingerdarmgeschwür 72: 299 Geiger counter experiments 81: 122

Granulom, eosinophile 80: 9 grosse tubérosité de l'estomac 97: 57 (L)

groupes sanguine et maladies de l'estomac 82: 238 (E)

Herz Beziehungen zwischen - und 81: 177 (E)

hyperventilation test in gastroduo-denal and neurocirculatory dystonia 79: 294

mucosa, intestinal metaplasia 86: 353 malformations 78: 400 (A)

en cascade 78: 320 (E)

Ektopie, kongenitale 77: 93

ptose 76: 317 (A)

lues 78: 402 (A); 82: 233 (C) lymphogranulomatose de Г- 76: 317 (A); 86: 531 , Neoplasmata (v. carcinoma) 70: 100 (A); $72: 351$ (A); $73: 123$ (A); $74: 317$ (A); 76: 310 (A); $78: 398$ (A); 80: 247 (A); 81: 189 (A); 82: 59 (A)

-, adénome de la petite courbure 71 :

321 (C) -, Magenlipom 90: 353 -, neurogene Magentumoren 79: 214 polypose gastrique et anémie de Bier-mer 98: 170 sarcoma 76: 310 (A) -, primäre 87:

299 -, Chirurgie 97: 57 (L) tumeurs bénignes de l'estomac et pré-cancer 86: 508 am Magen, gehäuftes gleichzeitiges

Auftreten gutartiger - 72: 60 (C)

Occlusion médio- transverse par mé-gacôlon d'origine mécanique 67: 55 (C)

oedema of stomach, in case of familiar Quincke's disease gastropically verified 65: 129 
operatus (v. Gastrectomie, Therapia, Chirurgia) 74:184 (L), 298 (A), 377 (L) 76: 299 (A); 80: 190 (A), 248 (A); 82 315 (L); $85: 121$ (A); $87: 332$ 88: 267 (A) , Karzinom des Restmagens 77: 281,

396 -, Magenoperationen und Fettresorp-

tion 98: 157 -, Fistula gastro-jejunocolic 82: 285;

87: 383; 89: 43 gastro-entérostomie, cancer du ver-sant gastrique 79: 41 (C)

for duodenal ulcer, results of $-83: 41,43,45$

for duodenal ulcer. Late results 83: 51

operation on the stomach, haemato-logical and nutritional effects 79: 337 -, -, nächtliche Sekretion 83: 167 postoperative Gastritis, gastroskopi-sche

Untersuchungen 67: 309

Magenbeschwerden 69: 286

sutures persisting casting off into the stomach after - 96: 291

pepsinogenes Serum bei Kranken mit Leberzirrhose 91: 71 Perigastritis deformans, Klinik und Bedeutung 79: 65

Index Гerum ad vol. 64-100

309

Stomachus

-, Physiologia (v. Physiopathologia) 65: 286 (A); 66: 96 (A), 235 (A); 68: 74 (A); 69: 96 (A); 71: 175 (A); 73: 111 (A); 74: 163 (A); 76: 18, 109 (A); $78: 322$

(A); 80: 244 (A); 81: 182 (A); 91: 216 (A); 94: 190 (A) -, tiotricité normale et pathologique

analysée par la gastrographie

77: $231-,-$, aspects neuro-chimiques reflexes

78: 17 -, Magenmotorik und Magenschleim-

fraktionen 80: 340 -, Motrizität, Einfluß der Absorption

durch Zwölffingerdarm auf - 65: 72 Mucosa 76: 195; 85: 140 -, Magenschleimhautzelle, Versor-

gung mit wasserlöslichen Gasen

85: 306 -, does duodenal content exert a

harmful effect on gastric - ? 98: 233 -, gastric secretion and parietal cells

99: 319 -, intestinal metaplasia of - 86: 363 -, protection against acid-pepsin.

Urea-urease mechanism as factor

in the depression of gastric acidity

76: 86 pression intra-gastrique des sujets normaux 93: 46

Protease des Magens. Uber die Art der in der schwach sauren Zone wirk-samen - 81: 44

- $\quad$ du sue gastrique. Etude physico-

chimique et clinique des deux -

77: 193

-, Kathepsins, Biologie des - 73: 320 (L); 76: 63, 255; 77: 33, 145

secretion gastrique, aspects anatomo-

fonctionnels 80: 37

-, hormonal control 76: 82

-, acid by the stomach, mechanism 76: 78

H-Ionenkonzentration im Magen wäh-

rend der Verdauung 81: 284

- $\quad$ sues gastrique. Etude immuno-élec-

trophorétique 94: 236

Physiopathologie (v. Physiologia) 82:

54 (A); 83: 187 (A)

-, ballonnement épigastrique, note

sur mécanisme 65: 236 folic acid deficiency: its effect on the gastro-intestinal tract 89: 347 -, gastric acidity: Urea-urease mechanism as factor in depression of -76: 86 -, -, secretion and - in duodenal ulcer, basal and histamine-sti-mulated 96: 351; 97: 39 -, - - in response to Insuline. Hypo-glycaemia in Heidenhain pouches in dogs 78: $364-,-,-$, influence of pituitary and

adrenal glands 85: 205

-, Magensaft: Rhodan-Eiweiß-Rela-

tion im Speichel für Nachweis pa-

thologischer Eiweißkörper 85: 313

-, Magenfunktionen, psychische Be-

einflussung 81: $167-,-$, , Saftsekretion nach Typhus ab-

dominalis 64: 44 -, -, Beziehungen zwischen Säureaus-scheidung und Bakterienflora 94: 315 -, -, Schleimund Ulcusgenese 74:134 -,-,--haut bei Leberkranken

90: 373 -, resistance to digestion of stomach-implanted dog's colon 83: 58 -, Uro-Pepsin, Uro-Kathepsin Be-ziehung zum histologischen Zu-stand der

Magenschleimhaut 91: 285 -, Hypoproteinämie bei - 96: 12 prolapsus de la muqueuse gastrique à travers le pylore 76: 227 (E); $78: 403$ (A) 
Magenschleimhautprolapses, Bei-träge zur Frage 79: 27; 100: 63 (L)

pylorischen trans-Schleimhaut-prolapses, röntgenologische Er-scheinungsform 87: 19

Polyps: diagnosis and treatment

86: 375

pylorus

-, antrum 82: 330

310

Index rerum ad vol. 64-100

Stomachus

,-- , stenoses non néoplasiques de l'a-dulte 98: 360

,,--- , congenital and human genetics 97: 50 (E)

-, Radiologie des klinischen Magens 95: 135 (L)

,-- , diagnosis of gastric atrophy 85: 216

,,-- rapport entre lesions histologiques et signes - des cancers de Гestomac au debut 77: 73

,-- precancerous gastropathies $86: 374$

-, Sarcoma (v. Neoplasmata)

-, stases 76: $61(\mathrm{~L})$

-, Therapia 65: 109 (A); 66: 101 (A); 68: 77 (A); 69: 110 (A); 74: 247 (A); 81: 189 (A)

-, -, ACTH-Vitamin-C-Gaben kombi-niert auf Magensekretion nach Hi-stamin 77: 299

-, -, Motilítät des Magens, Wirkung von BZ 55 und D 860 auf die Azidität 87: 65

-, -, Chirurgie (v. Stomachus operatus, Gastrectomie) 70: 365 (A); 81: 358 (A); 82: 60 (A); 83: 200 (A); 85:120 (A); 87: 61 (A); $91: 242$ (A); $93: 123$ (A); $94:$ 197 (A)

-, -, chlorhydrate d' $\alpha$-phényl- $\alpha$ (2-dié-thylaminoéthyl) glutaminide, influence sur secretion gastrique et mobilité gastro-intestinale 90: 29

-, -, Ephedrins, Wirkung des - auf die Pepsinogen-Aktivität des Plasmas 94: 182

,-- , inhibiteurs de la mono-amino-oxy-dase. Etude sur la secretion gastrique de Гantagonisme entre réser-pine et - 98: 217

-, -, Pharmaka, inhibitorische Wirkung 78: 269

-, -, Kaffee-Bohnen und Kaffee-Ersatz-mittel, vergleichende Wirkung 68: 182

-, -, «Magentag» im Spiegel der Säure-sekretion 83: 177

,,-- mercury salts, effect on gastric secretion 99: 105

-, Mersalyl (Mercurgan), inhibition of

gastric acid with - 86: 381 -, parasympathetic blocking agent, effect on gastric secretion and gastrointestinal motility 82: 225 -, «Pulvis stomachicus

Birckmanni»,

aus dem 16. Jahrhundert 84: 169 -, reserpine effect upon basal gastric

secretion 90: $401(\mathrm{~T})$-, post-réserpiniques, lesions - 98:

225; 99: 267 (T) antirheumatische Präparate, für den Magen gefährlich 85: 50 (E) -, Robadin, Einfluß auf Magensaftei-

weiß 90: 21 -, Sulphonamid and antithyroid

drugs, on gastric secretion 92: 55 Tuberculose (v. Gastrectomia) 71: 239 (A); 76: 316 (A); 78: 402 (A) Volvulus 69: 119 (L); $94: 379$

Succinic dehydrogenase of human stomach in patients with peptic ulcer or carcinoma 98: 376

Surgery: v. Chirurgia

Surrenal 64: $152(\mathrm{R}) ; 68: 335(\mathrm{~A})$

-, Beziehungen zwischen - und Ulkus-

krankheit 76: 169 -, Insuffizienz und ihr Formenkreis 67:

167 (L)

Sympathektomie, periarterielle - bei schwerer Hepatitis 96: 203

Teeth: v. Dentes

Therapia

-, progrès recent en gastro-entérologie

74: 161 (E) - der Verdauungs- und Stoffwechsel-

krankheiten 75: 64 (L) -, Fortschritte der Arzneimittelfor-

schung 100: $134(\mathrm{~L})$

Thioctique, acide -, biocatalyseur hépa-totrope dans le coma hépatique 89: 131 (T)

Thiopropérazine, dans traitement de l'ulcère gastro-duodénal 100: 61 (T)

Index rerum ad vol. 64-100

311

Thrombopénie, role dans les hémorra-gies des cịrrhoses alcooliques 99: 402 (L) Thrombocytémie essentielle 68: 148 Tubage duodenal: v. Choledocus, Pancreas, Vesica fellea 
Typhus abdomịalis, perforations digestives de la fièvre - 66: 56 (L) -, Magensaftsekretion nach - 64: 44 -, Dauerausscheider, bakteriologische Untersuchung der Duodenalsonden-flüssigkeit 94: 1

Thyreoïdea 64: $150(\mathrm{R})$; 66: $112(\mathrm{~A})$;

68: 334 (A) -, insuffisance, troubles gastro-intesti-

naux 74: 225 -, thyroxine, métabolisme dans Thépa-

tite épidémique 87: 129

Tolbutamide in cirrhosis of the liver 98:

181 (T) Trace elements in serum of gastrecto-

mized patients 95: 374 Trichloracetic acid, universal chemical

agent 76: 285 «Tris», bloqueur ganglionnaire; phar-

macologie gastroscopie 90: 101 Tropenkrankheiten, Lehrbuch der -

100: 207 (L)

Tuberculose

des gastrectomisés, facteurs pathogé-niques 84: 17

pulmonaire et ulcère gastro-duodénal 95: $130(\mathrm{E})$

ulcéreuse iléo-cæcale, indications ac-tuelles du traitement chirurgical 80: 129

-, clinical picture of primary gastrointestinal - 99: 359

-, pulmonary following partial gastrec-tomy 81: 91; 90: 65

-, sanatorium, basal uropepsin excretion rate of patients in a - 89: 93

-, Lungen, Kombination von Ulkus und - 98: 299

Tumeurs: v. Carcinoma, Neoplasmata Tumor: v. Carcinoma, Neoplasmata

Ulcerative colitis: v. Colitis ulcerosa

Ulcus pepticum

64: 169 (L), 267; 65: 287 (A); 67: 281; 68: 84 (A); 70: 97 (A); 72:145; $73: 117$ (A), 273 (E); 74: 186 (L); 76: 120 (A); $77: 327$ (L); $78: 406$ (L); $80: 184$ (A); 84: 272 (L); 86: 58 (A), 436; 87: 55 (A); $88: 62$ (E), 71 (L), 265 (A); 91: 279 (A); 93: 62 (A); 94: 191 (A); $97: 58$ (L)

$\mathrm{ABO}$ group secretion in - families $92: 99$

accidents, médecine des - et ulcus 67: 1

acid in test meals, influence of low concentration 86: 506 acute -, prognosis causing haemorrhage 78: 369

hypoalbuminemia in - 99: 225 alimentation en période de guerre, recrudescence - 70: 225 Altersulkus an Magen und Zwölffin-gerdarm 75: 63 (L)

anaphylactoid -, «foreign» juice effect in gastric mucosa 99: 230 Antrum gastritis und peptisches Ge-schwür 90: 110

atophan, ulcer prevention 86: 493 biliaires, syndromes ulcéro- 82: 19 brûlures cutanées, - consécutif à 70: 57

et carcinoma 78: 381 (A) -, recherches anatomo-cliniques 75:

321 -, dégénérescence maligne de 1'-, biop-sie exfoliative dans le diagnostic 86: 521 (esp.) -, Differentialdiagnose zwischen Ulkus und Magenkarzinom 77: 129 -, differential diagnosis, therapeutic test of benign and malign - 92: 1 -, - des präpylorischen - 73: 352 -, Gastritis und - 67: 110 (L); 77 : 328 (L)

312

Index rerum ad vol. 64-100

\section{Ulcus pepticum}

-, in surgically resected stomachs 95: 341 -, Häufigkeit des - des Magens

71: 210 -, histologie des stades initiaux du

carcinome au bord de $\Gamma$ ulcère

99: 81 -, 1'ulcère gastrique prédispose-t-il

au cancer? (ital.) 86: 376 -, risk of gastric cancer after medical

treatment for gastric ulcer $88: 1$ - ulcères transformés, ulcères bénins

et cancers ulcériformes 64: 259 Clinique 68: 84 (A); 71: 182 (A); 73: 182 (A), 243 (A), 302 (A); 76: 229 (A); $78: 375$ (A)

-, unkomplizierte Ulkus 87: 30 -, pains in peptic ulcer patients and

its significance for the diagnosis and

therapy 87: 105 -, medical disorders relation of peptic

ulcer with other - 76: 325 coronary sclerosis, relationship of chronic - 85: 201; 89: 292, 301 Diagnose von peptischen Geschwüren, Wert der Fadenprobe 64: 65 -, early detection of ulcerous disease

91: 245 -, gastroskopisches, neues Symptom

beim Zwölffingerdarmgeschwür 72:

299 -, Gastroskopie und Pathologie des

Magengeschwürs 93: 396 -, méthodes nouvelles dans le - de la

maladie ulcéreuse 86: 517 -, Salzsäureprobe in - 74: 270 -, Registrierungssystems, Darstel-

lung eines neuen eigenen - 80: 231 Dienstbeschädigung, Magenentzün-dung und Magengeschwür 67: 109 (L) Duodenalsaft bei - $99: 343$ duodeni 83: 247 (A) experimentelles Magengeschwür 78: 295 (R) -, Ulkuserzeugung und therapeuti-

sche Beeinflussung mit Robuden 
78: 11

-, antidiabetic Sulfonyl compounds

92: 86 -, atophan ulcer experiments aiming

at prevention of $-86: 493$

ulcerogenic effect of Cortisol in the rat 98: 100

Shayschen Ulkus bei Ratten, Wir-kung der Duodenum-Alkalisierung auf die Entwicklung des - 98: 108

Shay rat, effect of the new corti-coids, Dexametasone and Triam-cinolone on the -97: 75

geographical study 65: 1

giant gastric ulcer 76: 89 , Glucids

-, glycémie au cours de la maladie ulcéreuse 73: 209

-, carbohydrates metabolism and peptic ulcer 99: 394

-, hypoglycemia, etiologic factors in causation of abdominal pain in peptic ulcer and - 86: 535

bénin de la grande courbure, aspect

néoplasique 64: 282 , große Magengeschwiire, röntgenologi-

sches und klinisches über - 71: 142

Haemorragia 76: 232 (A); 78: 377 (A)

- digestive et aspirine 91: 274 (E)

, -, diagnostic value of gastric aspiration in - 79: 355

, - gastric, prognosis of acute - 78: 369

, -, management of massive - from upper gastro-intestinal tract 77: 1

,- ulcéreuses, résultats et indications du traitement chirurgical 93: 258 (T)

, - gastro-duodénales d'origine ulcéreuse, notions étiologiques sur les -95: 396 (E)

,- digestives, hyperazotémie dans -64: 208

, Histamine, role in production of peptic ulcer 86: 489

, Hypophysenvorderlappen und Magengeschwür 82: 162

, incidence of peptic ulcer in Denmark 80: 64 (L)

Index rerum ad vol. 64-100

313

\section{Ulc $\alpha \beta$ peptîcum}

,-- , changing - of peptic ulcer of the

stomach and duodenum 66: $116(\mathrm{~L})$-,

individual 81: $361(\mathrm{~L})$

insuffisance hépatique et - 83: $100 \quad-$,

Insuloma et - 93: 157

-, nésoblastome et - 94: 187 (E) -.

Jejuni 74: 302 (A)

-, postoperativum 87: 369

après gastrectomie 79: $148 \quad-$ -

Kathepsinbestimmung, Uro -, klini-

sche Bedeutung 84: $283 \quad-$,

komplizierte - 87: 95

mécaniques, role des facteurs - dans -. la genèse de - 67: $168(\mathrm{~L})$

- médicamenteux chez le rat 94: 273,

337; 98: 257

-, - à la phénylbutazone 91: 109

,-- et cortisone 91: 182

-, - et corticothérapie 91: 124 (E) -, -, poussée evolutive par réserpine 92: 181 (T) mixtes gastro-duodénaux 88: $130(\mathrm{~L})$

-, Nebenniere, Beziehungen zwischen -.

Ulkuskrankheit und - 76: 169-,

-, neuro-chemische Magenreflexe, Zwölf-

fingerdarm, neues eigenes Registrie-

rungssystems 80: 231 -.

-, occupational factors in aetiology of gastric and duodenal ulcers (v. profession) 79: 63 (L)

-, Ovariafunktion in Geschwiir-Krank--, heiten 93: 294

-, pancreatic heterotopic tissue, chronic -. peptic ulceration 92: 73

-, parathyroïdien, fonctionnement dans 
98: 193

-, Pathogénie 71: 179 (A); 72: 270 (A);

73: 117 (A); 74: 170 (A); 76: 124 (A);

78: 329 (A) -, Pepsinaktivität in Geschwür-Krank-

heit 93: 79 -, Perforation 76: 234 (A) -, -, Behandlung, konservativen 80:

$347-,-$, problèmes du traitement 75: $61(\mathrm{~L})$-, -, -, traitement non-opératoire 72: $260(\mathrm{E})$

-, traitement de l'ulcère - haut sítué

de la petite courbure 80: 94 periodischer Verlauf des -, ist er sai-sonsgebunden ? 89: 51 (E) professions traumatisantes dans genèse - 67: 30

pyloric antrum and - 89: 282 radiologie, diagnostic difFérentiel des ulcérations gastriques 86: 126 (L) -, «nichę signe de cicatrisation de -

84: 193 (E) Schleim, Magen- und Ulkusgenese 74: 121, 134 Schwankungen der Manifestation des

in Friedens-, Kriegs- und Nach-

kriegszeit 75: 147

SchutzstofFmangels, das Ulkus als Problem eines - 81: 364 (L) secretion, basal and histamine stimulated and acidity in - 96: 351 ; 97: 39

Sténose du cardia par cicatrice d'ulcus 65: 277 (C) pylorique 80: 247 (A); 94: 196 (A)

succinic dehydrogenase of human

stomach in patients with peptic ulcer

or carcinoma 98: 376

Therapia 67: 110 (L); 69: 188; 71: 186 (A); 72: 279 (A); 73: 121 (A), 343; 74: 247 (A), 254 (L); 75: 113 (E); $82: 64$ (L); $96: 332$ (E)

-, prevention de la rechute 100: 63 (T) -, Kinetik der Ulkusheilung 89: 160 -, Zelluntersuchung des Magensaftes,

diagnostische Bedeutung für -71 :

283,289

Chirurgicalis (v. Gastrectomie, Stomachus operatus) 71: 190 (A); 72: 361; 74: 253 (A); 75: 34 (T); 76: 244 (A); $78: 386$ (A); $85: 63$ (L); $88: 51,94$ -, follow-up of - 93: 15

86: 60 (A)

Gastrectomie 68: 286; 84: 84 (A);

,-- fundique $81: 51(\mathrm{E})$

,-- avec resection de l'ulcère haut

situé de Гestomac 80: 58 (E)

- $\quad$-, - polaire supérieure 80: 84

314

Index rerum ad vol. 64-100

Ulcus pepticum

-, -, Resektionstechnik 85: 127 (L) -, gastroenterostomy, vagotomy

and partial gastrectomy 89: 307 -, gastroscopic selection of cases

for gastroenterostomy 83: 54 -, haut situés de la petite courbure

de Testomac 80: 65 -, results of comparison of partial

gastrectomy with gastroenterostomy in - 89: 304 -, late results of vagotomy and

gastroenterostomy in treatment

of chronic - 89: 311 -, Vagotomy 72: $386(\mathrm{R}) ; 73: 240$;

74: 238 (E), 280, 306 (A); 79:

$194-,-$, place actuelle de la - en chir-

urgie gastrique 76: 108 (E) -, - oder Splanchnicotomie zur -

74: 156 Diaetetica, schnelle Rückbildung großer Ulkusnischen unter - 64: 1 -, almonds sweet dried in - 86: 505;

91: 315 -, carbohydrate for - 86: 496 -, Sondenbehandlung chronischer

Magen- und Zwölffingerdarmge-

schwüre 82: 63 (L) - «Vitamin-U»-Therapie der Ma-

gendarm Ulcera, gegenwärtiger

Stand der sog. - 84: $119(\mathrm{R})$-, -, sue de choux et de banane

82: 308 (T) Medicalis 69: 2; 74: 251 (A); 76: 236 (A); 78: 382 (A); 81: 255 (A) -, almonds in ulcer therapy 86: 505;

91: 315 -, aluminium-dehydroxy -, antacid effect and use in peptic ulcer

91: 214 (T) -, Antrenyl Duplex, Anwendung

beim - 90: 39 -, konservativer, Dauerergebnisse

von Zwölffingerdarmgeschwür

86: 100 -, Bismuthbehandlung 66: 1

échecs, quelques causes des -

dans traitements - 73: 327 
Einfrieren des Magens zur -

100: 65

enzymes inhibitors, in - 86: 485

pepsin inactivation in -70 :

$53(\mathrm{~T})$

Polysaccharids, das die pepti-

sche Proteolyse beim Magen-

und Duodenalgeschwür hemmt

96: 85; 100: 63 (T)

Gynergen und Dihydroergota-

$\min 72: 135(\mathrm{E})$

hormones 69: 31

Keimdrüsenhormonen 66:

343 (R)

Kritik der internen Ulkusthera-

pie 64: 198

mucine, recherche expérimen-

tale sur Faction d'une nouvelle preparation - (ital.) 78: 283

novocaïnique, par infiltration du cerveau préfrontal 82: $181(\mathrm{~T})$

nouvelles medications 100:

129 (E)

pantothénique, acide, dans - 94:

366

effets des - parentéraux sur cholinestérase des ulcéreux 71: 273

placebo effect in peptic ulcer and

other gastroduodenal disorders

94: 11

radiothérapie abdominale et ul-

cère face antérieure de Гestomac

91: 35

-, gastric, in the treatment of

duodenal ulcer 87: 345 (T) Reflexbehandlung, nasale, des Ulcus ventriculi und duodeni 78: 44

résines synthétiques dans $-75: 112(\mathrm{~T})$

Robuden 73: 157; 80: 294; 81: 257

sérothérapie anti-ulcéreuse 73: 90(E)

Succus liquiritiae, Ergebnisse bei - 81: 24

Index rerum ad vol. 64-100

315

\section{Ulcus pepticum}

-, - -, unspezifischen -, neue Gesichts-punkte 78: 227

- $\quad-$, Thiopropérazine 100: 61 (T) (T)

tuberculose pulmonaire et -95 :

130 (E) (v: Tuberculose)

Unfallserkrankung (v. accident) 64:

290

vascularisation de $\Gamma$ estomac ulcéreux 72: 154

of the stomach in relation to the

aetiology of $-81: 66$

-, problème anatomo-radiologique du duodenum et le siege de Гulcus bulbaire 70: 110 (L); 74: 186 (L)

ventriculi 81: $184(\mathrm{~A}) ; 82: 56(\mathrm{~A}) ; 83$ :

193 (A); 84: 79 (A); 85: 119 (A)

-, virus in the fibres of vagus nerve as probable cause of recurrence in gastro-duodenal ulcerated persons 77: 48

Urographie descendante dans diagnostic d'un «abdomen aigu» 86: 53 (E)

Uropepsin 
studies in gastroenterology, clinical applicability 86: 464

excretion, influence of sex and age 89: 93

Urtikarieller Krankheiten, Behandlung mit K-Vitamin 71: 171 (R)

Vaccinetherapie der Colitis ulcerosa 68: 21

auto - spezifischen und Enterokok-ken-Serumtherapie in der Behandlung der Colitis ulcerosa 68: 36

- von Magensaft in der Behandlung

des Magenkrebses 73: 45

Vateri, papilla: v. Choledocus Verdaulichkeit von Kartoffelbrot

69: 117 (L) Verdauung: v. Digestio, Gastroenterolo-

gia

Venography, costal intra-osseous, in diagnosis portal hypertension 88: 21

Vesica fellea (v. Choledocus, Fel, Icterus)

64: 59 (L); 75: 272 (L); 77: 64 (L); 94: 270 (L); 98: 127 (L), 394 (L) 100: 207 (L)

carcinome of gall-bladder 79: 357 Cholécystectomie -, complications médicales de la - 67:

331 (L) -, experimental effect on resorption

of isij-labelled fats 99: 301 -, Folgezustände nach - und Behandlung 76: 149 -, Mißerfolge und Beschwerden nach

- 85: 127 (L) -, physio-pathologie de la - 69: 150 -, post - syndrome 83: 27; 89: 321;

93: 288 Cholecystitis 81: 356 (A); 83: 372 (A); 89: 377 (A)

infections chroniques par brucel-, lose de Bang 76: 1

du point de vue chirurgical 69: 177 Cholecystokinin, tests of gall-bladder function in man 78: 349; 95: 100 Cholelithiasis 81: 356 (A); $83: 373$ (A); 89: 378

(A); 84: 330 (L) -, formation of gall-stones 83: 18 -, --, experimental studies on the colloid-chemical mechanism 80: 313; 85: 271 -, sphinctérotomie transduodénale

dans traitement lithiase biliaire 99 :

267 (T) Gallenblase, fiber eine neue Möglich-keit der - Diagnostik 96: 184 Dyskinésies 75: 386 (A); 76: 379 (L); 83: 125 (E), 371 (A) Fistules biliaires 76: 37

(A); 89: 380 (A) - anastomoses bilio-digestives 99:

78 (L) gastric dyspepsia, studies on functional disorders of the biliary tract in -85: 290

316 Index rerum ad vol. 64-100

Vesica fellea

Ileus biliaire 76: 37 (A)

histological changes of liver in diseases biliary tract 91: 221

Pneumocholecystítis, akute 76: 350

Röntgen 69: 326 (A); 89: 376 (A); 96:

246

-, cholangiocholecystography by N. H. Aldrige, advantages and disadvantages 85: 27

-, - laparoscopique 83: $110(\mathrm{R})$

-, cholecystangiography 83:11

-, cholecystography, combined, and barium meal examination 89: 330

-, - orale oder intravenöse 88: 334

-, radio-manométrie trans-hépato-vé-siculaire 77: 53 (E)

-, 2 Richtungsebenen, Untersuchung der Gallenblase 84: 143

-, transhepatic cholangiography 93: 65

séquelles vésiculaires de $\Gamma$ hépatite

épidémique 71: 295 (C)

Stippchen-Gallenblase, Problem der -

98: 256 (L)

Tubage duodenal

- minute 77: $175(\mathrm{E}) ; 87: 241 ; 88: 361$

-, Bakterienbefund in Duodenalson-dedflüssigkeit 91: 20; 92: 267

-, chromodíagnostic by biliary intubation and methylene Blue 93: 210

-, FarbstofFelimination durch die Gal-le, Wirkung von Decholin auf die -96: 171

-, Fermentbestimmung im Duodenal-saft 100: 209

Virus in vagus nerve, as cause of recurrence in gastro-duodenal ulcers 77: 48

Vitamines 64: 252 (R); 66: 52 (A); 68: 308 (A), 320 (A); 70: 359 (A); 71: 171 (A); 75: 63 (L)

A, metabolism, thyrogeníc osteoporosis and arthrosis relationship with - 65: 51

-, Resorption beim Dumping-Syn-

drom 95: 34

B12 in Beziehung zum histologischen Bild der Magen-Fundusschleímhaut 84: 103, 273 
- à doses massives dans le traitement

des cirrhoses du foie 82: $365(\mathrm{~T})$

-, effect on liver regeneration after

partial hepatectomy 100: 75

et carences alimentaires 70: 54 (L)

-, gegenwärtige Vitaminversorgung 68: 168 (L)

- $\quad \mathrm{K}$ in Behandlung von urtikariellen

Krankheiten 71: $171(\mathrm{R})$

- , klinische Anwendung 65: 308 (L)

und Hormone 64: 169 (L)

$\mathrm{U}$ (facteur anti-ulcus), traitement des ulcères gastro-duodénaux par le sue de choux et de banane 82: $308(\mathrm{~T})$

-, Therapie der Magendarm-Ulcera

84: 119 (R)

Voies biliaires: v. Choledocus

Water: v. Aqua

Wasser: v. Aqua

Whipple, morbus: v. Steatorrhea

Xanthoma pseudo elasticum, haemate-mesis due to - 89: 345

Zahn: v. Dentes

Zuekerkrankheit: v. Diabetes mellitus Zwerchfell: v. Diaphragma Zwölffingerdarm: v. Duodenum 